\title{
17. X-RAY MINERALOGY DATA FROM THE CENTRAL PACIFIC, LEG 33 DEEP SEA DRILLING PROJECT ${ }^{1}$
}

\author{
H.E. Cook and I. Zemmels, University of California, Riverside, California
}

\section{METHODS}

Semiquantitative determinations of the mineral composition of bulk samples, $2-20 \mu \mathrm{m}$, and $<2 \mu \mathrm{m}$ fractions were performed according to the methods described in the appendix of Volume 28.

The method of sample preparation, in brief, is as follows: Bulk samples are washed to remove seawater salts and are ground to less than $10 \mu \mathrm{m}$ under butanol. A portion of the sediment is decalcified in a sodiumacetate-buffered, acetic-acid solution $(p \mathrm{H} 4.5)$. The residue is fractionated into $2-20 \mu \mathrm{m}$ and $<2 \mu \mathrm{m}$ samples by wet-sieving and centrifugation. The $2-20 \mu \mathrm{m}$ samples are ground to less than $10 \mu \mathrm{m}$. These three preparations are treated with trihexylamine acetate to expand the smectites. All samples are X-rayed as random powders.

The results of the X-ray diffraction analysis are presented in Tables 1 to 14 . Tables 1 to 7 summarize the mineral data and provide stratigraphic information. The sediment ages, lithologic units, and nomenclature of the sediment types are from the DSDP Leg 32 hole summaries.

The complete list of samples from Leg 33 and their subbottom depths which were utilized in X-ray diffraction analysis are presented in Table 15. In a number of cases, two or more samples of similar lithology were composited. The composited samples are bracketed in Table 15. The samples are identified by their subbottom depths in Tables 1 to 14. The composited samples are identified by the subbottom depth of the stratigraphically uppermost sample of the composite.

The percent amorphous is a measure of the weight fraction of amorphous material in each sample, which commonly consists of biogenic silica, volcanic glass, palagonite, allophane, and organic material. The amorphous fraction is calculated from the total diffuse scattering of the sample. The method of calculation assumes that the diffuse scatter in excess of the diffuse scatter from the crystalline minerals is proportional to the amorphous content. The diffuse scatter of the crystalline minerals is determined from the mineral calibration standards (see Volume 28). Ideally, the amorphous fraction varies between 0 and $100 \%$, but, in cases where the minerals in the sample have a higher degree of crystallinity than the calibration standards, negative values can result. The negative values are reported as blanks; these samples can be assumed to contain little or no amorphous material.

The crystalline minerals are quantified by the method of mutual ratios using peak heights and concentration

'Institute of Geophysics and Planetary Physics, University of California, Riverside, California, Contribution No. 74-31. factors derived from the ratio of diagnostic peaks of minerals with the major peak of quartz. Unquantifiable minerals, i.e., unidentified minerals and minerals for which standards are not available, are tentatively quantified using a hypothetical concentration factor of 3.0 which is applied to the major peak of the mineral. The concentrations of the quantifiable minerals are summed to $100 \%$. The amorphous fraction and unquantifiable minerals are not included in the total. The unquantifiable minerals are reported on a qualitative scale as trace (less than 5\%), present (5\%-25\%), abundant (25\%$65 \%$ ), and major ( $>65 \%)$.

The precision of the mineral determinations is approximately \pm 1 weight percent of the amount present. Because of inevitable differences in the crystallinity of the mineral calibration standards and the minerals in the samples and also because of diffraction-peak interferences, the accuracy of the reported concentrations is often less than the precision of the method allows. In terms of the reported concentration, smectites may vary $\pm 50 \%$; micas, chlorites, cristobalite, tridymite, goethite may vary $\pm 20 \%$; and kaolinite, amphibole, augite, the feldspars, the zeolites, palygorskite, sepiolite, apatite may vary $\pm 10 \%$. Minerals which have stable crystal lattices and are not members of solid-solution series (or typically have limited crystal-lattice substitution in the sedimentary environment) such as quartz, lowmagnesium calcite, aragonite, dolomite, rhodochrosite, siderite, gibbsite, talc, barite, anatase, gypsum, anhydrite, halite, pyrite, hematite, magnetite will vary less than $\pm 5 \%$.

The user of the X-ray mineralogy data should bear in mind that (1) the reported values are not absolute concentrations and that some adjustment has to be made for the amorphous fraction and the unquantifiable minerals; (2) in a homogenous system of minerals, the mineral concentration trends are reliable because of the precision of the method, but when comparing mineral concentrations between different geographic regions or lithologic units additional information regarding the crystallinity of the minerals is required; and (3) the representativeness of the samples selected for X-ray diffraction analysis is the responsibility of the shipboard scientists and any questions pertaining to this aspect should be directed to them.

\section{MINERAL NOTES}

Twelve $<2 \mu$ m samples with a high smectite content from Hole 315A and Site 316 were tested for the presence of nontronite and beidellite using the GreenKelly method. The samples selected from Hole 315A were from 739.3, 779.2, 779.6, 788.5, and 932.7 meters; samples from Site 316 were from 515.4, 524.7, 533.6, 
TABLE 1

Summary of X-Ray Mineralogy Samples, Sample Depths, Lithology, Age, and X-Ray Diffraction Results, Site 314

\begin{tabular}{|c|c|c|c|c|c|c|c|c|c|c|c|}
\hline \multirow{2}{*}{$\begin{array}{c}\text { Sample } \\
\text { Depth Below } \\
\text { Sea Floor } \\
\text { (m) }\end{array}$} & \multirow[b]{2}{*}{ Lithology } & \multirow[b]{2}{*}{ Age } & \multicolumn{3}{|c|}{$\begin{array}{c}\text { Bulk Sample } \\
\text { Major Constituent }\end{array}$} & \multicolumn{3}{|c|}{$\begin{array}{l}2-20 \mu \mathrm{m} \text { Fraction } \\
\text { Major Constituent }\end{array}$} & \multicolumn{3}{|c|}{$\begin{array}{c}<2 \mu \text { m Fraction } \\
\text { Major Constituent }\end{array}$} \\
\hline & & & 1 & 2 & 3 & 1 & 2 & 3 & 1 & 2 & 3 \\
\hline $\begin{array}{l}0.0 \\
0-45^{\mathrm{a}} \\
0-45^{\mathrm{a}}\end{array}$ & $\begin{array}{l}\text { Brown } \\
\text { zeolitic } \\
\text { clay }\end{array}$ & Quat.? & $\begin{array}{l}\text { Clin. } \\
\text { Phil. } \\
\text { Phil. }\end{array}$ & $\begin{array}{l}\text { Magn. } \\
\text { Mica } \\
\text { Mica }\end{array}$ & $\begin{array}{l}\text { Goet. } \\
\text { Quar. } \\
\text { Quar. }\end{array}$ & $\begin{array}{l}\text { Clin. } \\
\text { Phil. } \\
\text { Phil. }\end{array}$ & $\begin{array}{l}\text { Magn. } \\
\text { Mica } \\
\text { Mica }\end{array}$ & $\begin{array}{l}\text { Goet. } \\
\text { Clin. } \\
\text { Quar. }\end{array}$ & $\begin{array}{l}\text { Mont. } \\
\text { Mont. } \\
\text { Mont. }\end{array}$ & $\begin{array}{l}\text { Cris. } \\
\text { Phil. } \\
\text { Mica }\end{array}$ & $\begin{array}{l}\text { Goet. } \\
\text { Mica } \\
\text { Quar. }\end{array}$ \\
\hline
\end{tabular}

${ }^{\mathrm{a}}$ Scrapings from outer drill collars.

TABLE 2

Summary of X-Ray Mineralogy Samples, Sample Depths, Lithology, Age, and X-Ray Diffraction Results, Hole 315

\begin{tabular}{|c|c|c|c|c|c|c|c|c|c|c|c|}
\hline \multirow{2}{*}{$\begin{array}{l}\text { Sample } \\
\text { Depth Below } \\
\text { Sea Floor } \\
\text { (m) }\end{array}$} & \multirow[b]{2}{*}{ Lithology } & \multirow[b]{2}{*}{ Age } & \multicolumn{3}{|c|}{$\begin{array}{c}\text { Bulk Sample } \\
\text { Major Constituent }\end{array}$} & \multicolumn{3}{|c|}{$\begin{array}{l}2-20 \mu \mathrm{m} \text { Fraction } \\
\text { Major Constituent }\end{array}$} & \multicolumn{3}{|c|}{$\begin{array}{c}<2 \mu \mathrm{m} \text { Fraction } \\
\text { Major Constituent }\end{array}$} \\
\hline & & & 1 & 2 & 3 & 1 & 2 & 3 & 1 & 2 & 3 \\
\hline $\begin{array}{r}1.3 \\
3.9 \\
57.9 \\
59.2 \\
61.9 \\
65.5\end{array}$ & $\begin{array}{c}\text { Unit 1: } \\
\text { Dark brown } \\
\text { nanno-foram } \\
\text { and } \\
\text { nanno-rad } \\
\text { oozes }\end{array}$ & 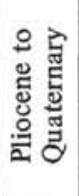 & $\begin{array}{l}\text { Calc. } \\
\text { Calc. } \\
\text { Calc. } \\
\text { Calc. } \\
\text { Calc. } \\
\text { Calc. }\end{array}$ & & & $\begin{array}{l}\text { Quar. } \\
\text { Quar. } \\
\text { Bari. } \\
\text { Bari. } \\
\text { Bari. } \\
\text { K-Fe. }\end{array}$ & $\begin{array}{l}\text { Mica } \\
\text { Mica } \\
\text { Mica } \\
\text { K-Fe. } \\
\text { Mica } \\
\text { Bari. }\end{array}$ & $\begin{array}{l}\text { Plag. } \\
\text { Phil. } \\
\text { Quar. } \\
\text { Quar. } \\
\text { Plag. } \\
\text { Plag. }\end{array}$ & $\begin{array}{l}\text { Mont. } \\
\text { Mont. } \\
\text { Mont. } \\
\text { Mont. } \\
\text { Mont. } \\
\text { Mont. }\end{array}$ & $\begin{array}{l}\text { Mica } \\
\text { Mica } \\
\text { Phil. } \\
\text { Plag. } \\
\text { Phil. } \\
\text { Phil. }\end{array}$ & $\begin{array}{l}\text { Quar. } \\
\text { Quar. } \\
\text { Mica } \\
\text { Quar. } \\
\text { Mica } \\
\text { Mica }\end{array}$ \\
\hline
\end{tabular}

562.9, 571.8, 610.5, and 771.2 meters (see Tables 10, 11, and 15 for sample identification).

The samples were saturated with lithium. Several samples were fractionated during centrifuging when it was evident that more than one population of clays was present. Randomly oriented mounts were prepared in duplicate. One set was heated for $12 \mathrm{hr}$ at $300^{\circ} \mathrm{C}$ and treated with glycerol; the other set was only treated with glycerol.

Most of the samples contained montmorillonite which expanded to $17-18 \AA$ and collapsed to $10 \AA$ with heating.

The sample from Hole 315A, depth 779.2 meters, was found to consist largely of montmorillonite. The coarser portion of the $<2 \mu \mathrm{m}$ fraction contained a large component of mica-montmorillonite mixed-layer clay.

The samples from Site 316, depth 610.5 and 771.9 meters, contained a portion which expanded after heating, indicating the presence of the nontronitebeidellite series.

Large quantities of montmorillonite were detected in numerous 2-20 $\mu \mathrm{m}$ samples in Holes 315A, 316, 317A. and 318. A microscopic examination of several $2-20 \mu \mathrm{m}$ samples revealed that they contained large quantities of birefringent, low-refractive-index aggregates which were presumed to contain the montmorillonite. The aggregates, which had survived the action of acetic acid in an ultrasonic bath, were also difficult to disperse in the microscope slide.

\section{DRILLING AND MUD USAGE}

Drilling mud containing montmorillonite and barite was used in Hole 315A between Cores 12 and 13 and Cores 18 and 19. An X-ray diffraction analysis of a sample of drilling mud from Leg 33 showed $88 \%$ montmorillonite, $5 \%$ barite, $2 \%$ quartz, $2 \%$ mica, and $2 \%$ feldspar. No samples were submitted from Core 13. The sample from Core 19 showed no traces of barite and no inordinate concentrations of montmorillonite.

\section{ACKNOWLEDGMENTS}

The writers wish to acknowledge the excellent work of David Berry in the interpretation of X-ray diffraction data, of Paul D. Johnson in X-ray data acquisition and data processing, and of Tom W. Halverson, Jr., in sample preparation. 
TABLE 3

Summary of X-Ray Mineralogy Samples, Sample Depths, Lithology, Age, and X-Ray Diffraction Results, Hole 315A

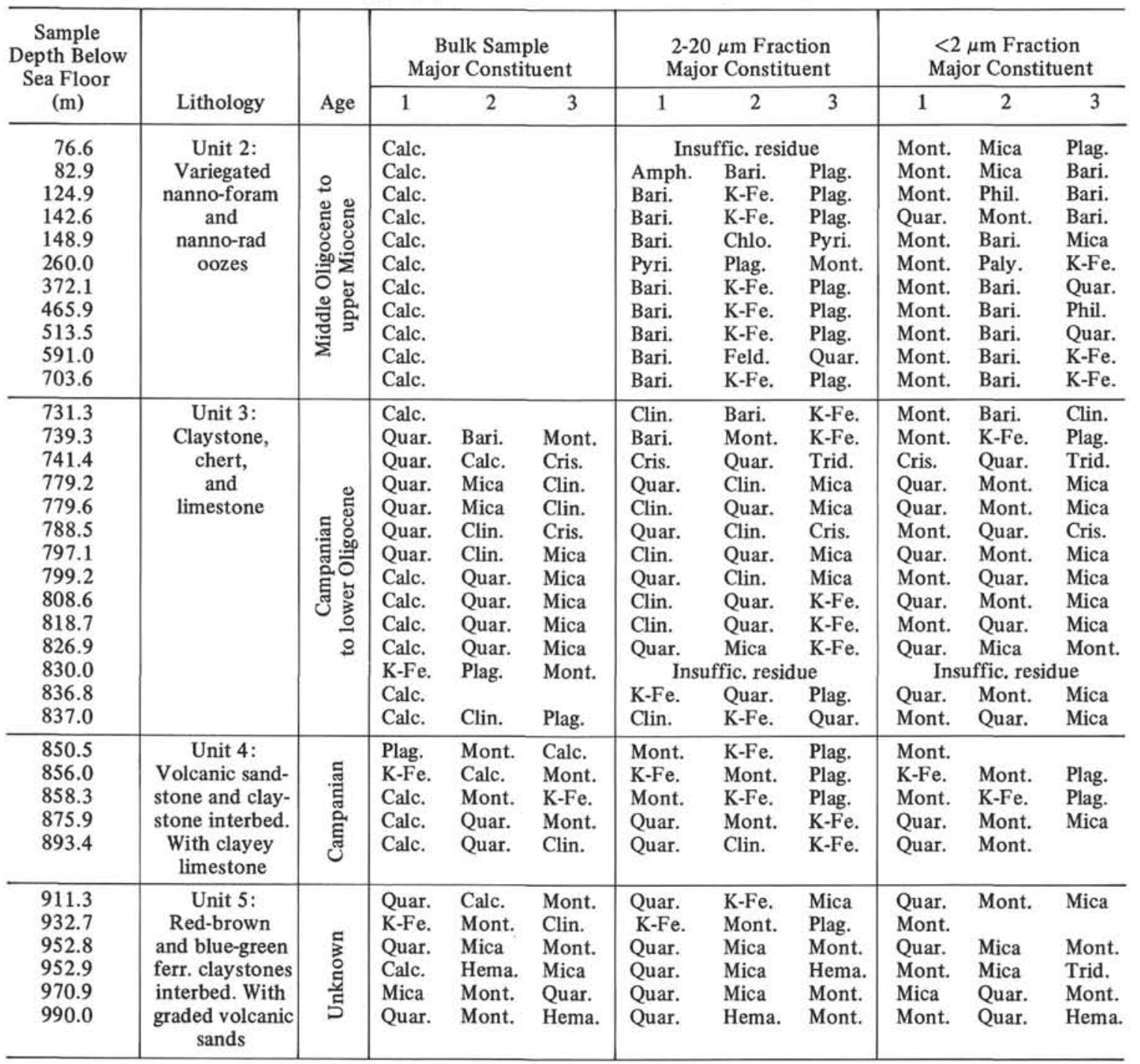


TABLE 4

Summary of X-Ray Mineralogy Samples, Sample Depth, Lithology, Age, and X-Ray Diffraction Results, Site 316

\begin{tabular}{|c|c|c|c|c|c|c|c|c|c|c|c|}
\hline \multirow{2}{*}{$\begin{array}{l}\text { Sample } \\
\text { Depth Below } \\
\text { Sea Floor } \\
\text { (m) }\end{array}$} & \multirow[b]{2}{*}{ Lithology } & \multirow[b]{2}{*}{ Age } & \multicolumn{3}{|c|}{$\begin{array}{c}\text { Bulk Sample } \\
\text { Major Constituent }\end{array}$} & \multicolumn{3}{|c|}{$\begin{array}{l}2-20 \mu \mathrm{m} \text { Fraction } \\
\text { Major Constituent }\end{array}$} & \multicolumn{3}{|c|}{$\begin{array}{c}<2 \mu \mathrm{m} \text { Fraction } \\
\text { Major Constituent }\end{array}$} \\
\hline & & & 1 & 2 & 3 & 1 & 2 & 3 & 1 & 2 & 3 \\
\hline 0.8 & Unit $1:^{\mathrm{a}}$ & a & Calc. & & & Bari. & Quar. & Plag. & Mont. & Quar. & Plag. \\
\hline $\begin{array}{r}2.7 \\
155.3 \\
268.3\end{array}$ & $\begin{array}{l}\text { Unit 2: } \\
\text { calc. and } \\
\text { siliceous oozes } \\
\text { and chalk }\end{array}$ & 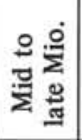 & $\begin{array}{l}\text { Calc. } \\
\text { Calc. } \\
\text { Calc. }\end{array}$ & $\mathrm{K}-\mathrm{Fe}$. & & $\begin{array}{l}\text { Bari. } \\
\text { Bari. } \\
\text { Bari. }\end{array}$ & $\begin{array}{l}\text { Quar. } \\
\text { Phil. } \\
\text { K-Fe. }\end{array}$ & $\begin{array}{l}\text { Plag. } \\
\text { Quar. } \\
\text { Plag. }\end{array}$ & $\begin{array}{l}\text { Mont. } \\
\text { Mont. } \\
\text { Mont. }\end{array}$ & $\begin{array}{l}\text { Quar. } \\
\text { Bari. } \\
\text { Quar. }\end{array}$ & $\begin{array}{l}\text { Mica } \\
\text { Plag. } \\
\text { Chlo. }\end{array}$ \\
\hline $\begin{array}{l}391.8 \\
448.6 \\
469.3 \\
486.5 \\
495.9 \\
515.4 \\
524.7 \\
533.6 \\
553.3 \\
562.9 \\
571.8 \\
573.6\end{array}$ & $\begin{array}{c}\text { Unit 3: } \\
\text { Siliceous chalk, } \\
\text { limestone, and } \\
\text { dolomite }\end{array}$ & 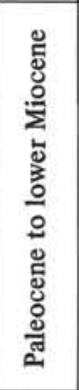 & $\begin{array}{l}\text { Calc. } \\
\text { Calc. } \\
\text { Dolo. } \\
\text { Calc. } \\
\text { Cris. } \\
\text { Calc. } \\
\text { Calc. } \\
\text { Calc. } \\
\text { Calc. } \\
\text { Calc. } \\
\text { Calc. } \\
\text { Calc. }\end{array}$ & $\begin{array}{l}\text { Clin. } \\
\text { Calc. }\end{array}$ & $\begin{array}{l}\text { Mont. } \\
\text { Trid. }\end{array}$ & $\begin{array}{l}\text { K-Fe. } \\
\text { K-Fe. } \\
\text { Clin. } \\
\text { Clin. } \\
\text { Cris. } \\
\text { Quar. } \\
\text { Quar. } \\
\text { Quar. } \\
\text { Quar. } \\
\text { Quar. } \\
\text { Quar. } \\
\text { Quar. }\end{array}$ & $\begin{array}{l}\text { Plag. } \\
\text { Plag. } \\
\text { K-Fe. } \\
\text { Bari. } \\
\text { Quar. } \\
\text { Mica } \\
\text { Mica } \\
\text { Mica } \\
\text { K-Fe. } \\
\text { K-Fe. } \\
\text { K-Fe. } \\
\text { Bari. }\end{array}$ & $\begin{array}{l}\text { Bari. } \\
\text { Quar. } \\
\text { Bari. } \\
\text { Quar. } \\
\text { Trid. } \\
\text { K-Fe. } \\
\text { Paly. } \\
\text { Bari. } \\
\text { Mica } \\
\text { Mica } \\
\text { Bari. } \\
\text { Mica }\end{array}$ & $\begin{array}{l}\text { Mont. } \\
\text { Mont. } \\
\text { Mont. } \\
\text { Cris. } \\
\text { Cris. } \\
\text { Mont. } \\
\text { Mont. } \\
\text { Mica } \\
\text { Mont. } \\
\text { Mont. } \\
\text { Mont. } \\
\text { Mont. }\end{array}$ & $\begin{array}{l}\text { Phil. } \\
\text { Mica } \\
\text { Quar. } \\
\text { Mont. } \\
\text { Trid. } \\
\text { Paly. } \\
\text { Paly. } \\
\text { Mont. } \\
\text { Mica } \\
\text { Mica } \\
\text { Mica } \\
\text { Quar. }\end{array}$ & $\begin{array}{l}\text { Plag. } \\
\text { K-Fe. } \\
\text { K-Fe. } \\
\text { Mica } \\
\text { Mica } \\
\text { Mica } \\
\text { Quar. } \\
\text { Quar. } \\
\text { Quar. } \\
\text { Quar. } \\
\text { Mica }\end{array}$ \\
\hline $\begin{array}{l}583.1 \\
584.4 \\
587.4 \\
609.8 \\
610.5 \\
613.6 \\
630.6 \\
638.4 \\
641.8 \\
642.1 \\
667.1 \\
668.1 \\
686.7 \\
704.5 \\
705.7 \\
727.0\end{array}$ & $\begin{array}{c}\text { Unit 4: } \\
\text { Volcaniclastic } \\
\text { breccia } \\
\text { and graded } \\
\text { sandstone, } \\
\text { chalk, and } \\
\text { limestone }\end{array}$ & 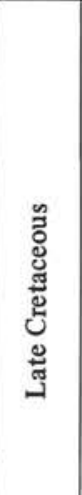 & $\begin{array}{l}\text { Calc. } \\
\text { Quar. } \\
\text { Calc. } \\
\text { Calc. } \\
\text { Calc. } \\
\text { Calc. } \\
\text { Calc. } \\
\text { Calc. } \\
\text { Calc. } \\
\text { Calc. } \\
\text { Calc. } \\
\text { Calc. } \\
\text { Calc. } \\
\text { Calc. } \\
\text { Calc. } \\
\text { Calc. }\end{array}$ & $\begin{array}{l}\text { Quar. } \\
\text { Mica } \\
\text { Quar. } \\
\text { Quar. } \\
\text { Mont. } \\
\text { Quar. } \\
\text { Mont. } \\
\text { Quar. } \\
\text { K-Fe. } \\
\text { Mont. } \\
\text { Mont. } \\
\text { Mont. } \\
\text { Quar. } \\
\text { K-Fe }\end{array}$ & $\begin{array}{l}\text { Quar. } \\
\text { Plag. } \\
\text { K-Fe. }\end{array}$ & $\begin{array}{l}\text { Quar. } \\
\text { Quar. } \\
\text { Quar. } \\
\text { Quar. } \\
\text { Mont. } \\
\text { Quar. } \\
\text { Mont. } \\
\text { Quar. } \\
\mathrm{K}-\mathrm{Fe} . \\
\mathrm{K}-\mathrm{Fe} . \\
\text { Mont. } \\
\mathrm{K}-\mathrm{Fe} \text {. } \\
\text { Quar. } \\
\mathrm{K}-\mathrm{Fe} \text {. } \\
\text { Ins } \\
\mathrm{K}-\mathrm{Fe}\end{array}$ & $\begin{array}{l}\text { Clin. } \\
\text { Clin. } \\
\text { Mica } \\
\text { K-Fe. } \\
\text { Clin. } \\
\text { K-Fe. } \\
\text { Quar. } \\
\text { Clin. } \\
\text { Hema. } \\
\text { Hema. } \\
\text { Clin. } \\
\text { Quar. } \\
\text { K-Fe. } \\
\text { Quar. } \\
\text { ffic. resi } \\
\text { Hema }\end{array}$ & $\begin{array}{l}\text { Mica } \\
\text { K-Fe. } \\
\text { K-Fe. } \\
\text { Mica } \\
\text { K-Fe. } \\
\text { Mica } \\
\text { Mica } \\
\text { K-Fe. } \\
\text { Clin. } \\
\text { Clin. } \\
\text { K-Fe. } \\
\text { Mont. } \\
\text { Mica } \\
\text { Mica } \\
\text { Pe }\end{array}$ & $\begin{array}{l}\text { Quar. } \\
\text { Mont. } \\
\text { Quar. } \\
\text { Mont. } \\
\text { Mont. } \\
\text { Quar. } \\
\text { Mont. } \\
\text { Quar. } \\
\text { Mont. } \\
\text { Mont. } \\
\text { Mont. } \\
\text { Mont. } \\
\text { Mont. } \\
\text { Mont. } \\
\text { Ins }\end{array}$ & $\begin{array}{l}\text { Clin. } \\
\text { Quar. } \\
\text { Mont. } \\
\text { Quar. } \\
\text { Mont. } \\
\text { Quar. } \\
\text { Mont. } \\
\text { K-Fe. } \\
\text { K-Fe. } \\
\text { Quar. } \\
\text { Quar. } \\
\text { Quar. } \\
\text { ffic. resic } \\
\text { Geot. }\end{array}$ & $\begin{array}{l}\text { Mica } \\
\text { Mica } \\
\text { Mica } \\
\text { Mica } \\
\\
\text { Mica } \\
\text { Mica } \\
\text { Mica } \\
\text { Plag. } \\
\text { Hema. } \\
\text { Mica } \\
\text { K-Fe. } \\
\text { Mica }\end{array}$ \\
\hline $\begin{array}{l}727.0 \\
744.8 \\
771.2 \\
830.3\end{array}$ & & & $\begin{array}{l}\text { Calc. } \\
\text { Calc. } \\
\text { Calc. } \\
\text { Calc. }\end{array}$ & $\begin{array}{l}\text { K-Fe. } \\
\text { K-Fe. } \\
\text { Mont. } \\
\text { K-Fe. }\end{array}$ & $\begin{array}{l}\text { Mont. } \\
\text { Plag. } \\
\text { K-Fe. }\end{array}$ & $\begin{array}{l}\text { K-Fe. } \\
\text { K-Fe. } \\
\text { Mont. } \\
\text { K-Fe. }\end{array}$ & $\begin{array}{l}\text { Hema. } \\
\text { Magn. } \\
\text { K-Fe. } \\
\text { Quar. }\end{array}$ & $\begin{array}{l}\text { Plag. } \\
\text { Pyri. } \\
\text { Quar. } \\
\text { Mica }\end{array}$ & $\begin{array}{l}\text { Mont. } \\
\text { Mont. } \\
\text { Mont. } \\
\text { Mica }\end{array}$ & $\begin{array}{l}\text { Geot. } \\
\text { K-Fe. } \\
\text { Quar. }\end{array}$ & $\begin{array}{l}\text { K-Fe. } \\
\text { Anal. } \\
\text { K-Fe. }\end{array}$ \\
\hline
\end{tabular}

${ }^{\mathrm{a}}$ Interbedded calcareous and siliceous oozes; Quaternary. 
TABLE 5

Summary of X-Ray Mineralogy Samples, Sample Depths, Lithology, Age, and X-Ray Diffraction Results Hole 317A

\begin{tabular}{|c|c|c|c|c|c|c|c|c|c|c|c|}
\hline \multirow{2}{*}{$\begin{array}{c}\text { Sample } \\
\text { Depth Below } \\
\text { Sea Floor } \\
\text { (m) }\end{array}$} & \multirow[b]{2}{*}{ Lithology } & \multirow[b]{2}{*}{ Age } & \multicolumn{3}{|c|}{$\begin{array}{c}\text { Bulk Sample } \\
\text { Major Constituent }\end{array}$} & \multicolumn{3}{|c|}{$\begin{array}{l}2-20 \mu \mathrm{m} \text { Fraction } \\
\text { Major Constituent }\end{array}$} & \multicolumn{3}{|c|}{$\begin{array}{c}<2 \mu \mathrm{m} \text { Fraction } \\
\text { Major Constituent }\end{array}$} \\
\hline & & & 1 & 2 & 3 & 1 & 2 & 3 & 1 & 2 & 3 \\
\hline $\begin{array}{l}554.2 \\
564.5 \\
576.9 \\
585.0 \\
602.4 \\
623.3 \\
644.1\end{array}$ & $\begin{array}{c}\text { Unit 2: } \\
\text { Foram-nanno } \\
\text { oozes and } \\
\text { chalk with } \\
\text { black chert }\end{array}$ & 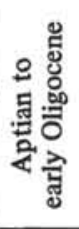 & $\begin{array}{l}\text { Calc. } \\
\text { Calc. } \\
\text { Calc. } \\
\text { Paly. } \\
\text { Calc. } \\
\text { Cris. } \\
\text { Calc. }\end{array}$ & $\begin{array}{l}\text { Clin. } \\
\text { Quar. } \\
\text { Quar. } \\
\text { K-Fe. } \\
\text { Cris. } \\
\text { Calc. } \\
\text { Mont. }\end{array}$ & $\begin{array}{l}\text { Mica } \\
\text { K-Fe. } \\
\text { Quar. } \\
\text { Quar. } \\
\text { Trid. } \\
\text { K-Fe. }\end{array}$ & $\begin{array}{l}\text { Clin. } \\
\text { K-Fe. } \\
\text { K-Fe. } \\
\text { K-Fe. } \\
\text { Cris. } \\
\text { Cris. } \\
\text { Clin. }\end{array}$ & $\begin{array}{l}\text { Quar. } \\
\text { Quar. } \\
\text { Quar. } \\
\text { Mica } \\
\text { Quar. } \\
\text { K-Fe. } \\
\text { K-Fe. }\end{array}$ & $\begin{array}{l}\text { K-Fe. } \\
\text { Mica } \\
\text { Mica } \\
\text { Quar. } \\
\text { Bari. } \\
\text { Trid. } \\
\text { Plag. }\end{array}$ & $\begin{array}{l}\text { Mont. } \\
\text { Quar. } \\
\text { Mica } \\
\text { Paly. } \\
\text { Cris. } \\
\text { Cris. } \\
\text { Mont. }\end{array}$ & $\begin{array}{l}\text { Mica } \\
\text { K-Fe. } \\
\text { Quar. } \\
\text { Mont. } \\
\text { Trid. } \\
\text { Trid. } \\
\text { K-Fe. }\end{array}$ & $\begin{array}{l}\text { K-Fe. } \\
\text { Mica } \\
\text { K-Fe. } \\
\text { Mica } \\
\text { Quar. } \\
\text { Mont. } \\
\text { Plag. }\end{array}$ \\
\hline $\begin{array}{l}651.0 \\
670.0 \\
698.6 \\
727.7 \\
755.9 \\
756.7 \\
757.4 \\
759.4 \\
777.7 \\
778.1 \\
779.6 \\
821.1 \\
849.9 \\
887.4 \\
887.4 \\
\end{array}$ & $\begin{array}{c}\text { Unit 3: } \\
\text { Volcaniclastic } \\
\text { sandstones and } \\
\text { siltstones }\end{array}$ & $\begin{array}{l}\text { E } \\
\text { 离 } \\
\text { है }\end{array}$ & $\begin{array}{l}\text { Mont. } \\
\text { Mont. } \\
\text { Mont. } \\
\text { Mont. } \\
\text { Mont. } \\
\text { Mont. } \\
\text { Mont. } \\
\text { Mont. } \\
\text { Mont. } \\
\text { Mont. } \\
\text { Mont. } \\
\text { Mont. } \\
\text { Mont. } \\
\text { Mont. } \\
\text { Mont. }\end{array}$ & $\begin{array}{l}\text { Plag. } \\
\text { Anal. } \\
\text { Sani. } \\
\text { K-Fe. } \\
\text { Hema. } \\
\text { Sani. } \\
\text { Sani. } \\
\text { Anal. } \\
\text { Sani. } \\
\text { Sani. } \\
\text { Sani. } \\
\text { Anal. } \\
\text { Anal. } \\
\text { Anal. } \\
\text { Anal. }\end{array}$ & $\begin{array}{l}\text { Clin. } \\
\text { Anal. } \\
\text { Plag. } \\
\text { Quar. } \\
\text { Anal. } \\
\\
\text { Sani. } \\
\text { Anal. } \\
\text { Calc. } \\
\text { Anal. } \\
\text { Sani. } \\
\text { Sani. }\end{array}$ & $\begin{array}{l}\text { Plag. } \\
\text { Mont. } \\
\text { Mont. } \\
\text { K-Fe. } \\
\text { Mont. } \\
\text { Mont. } \\
\text { Mont. } \\
\text { Mont. } \\
\text { Mont. } \\
\text { Mont. } \\
\text { Mont. } \\
\text { Mont. } \\
\text { Mont. } \\
\text { Anal. } \\
\text { Mont. }\end{array}$ & $\begin{array}{l}\text { Mont. } \\
\text { Anal. } \\
\text { Anal. } \\
\text { Mont. } \\
\text { Feld. } \\
\text { Sani. } \\
\text { Sani. } \\
\text { Sani. } \\
\text { Sani. } \\
\text { Sani. } \\
\text { Sani. } \\
\text { Sani. } \\
\text { Sani. } \\
\text { Mont. } \\
\text { Sani. }\end{array}$ & $\begin{array}{l}\text { Quar. } \\
\text { Sani. } \\
\text { Plag. } \\
\text { Hema. } \\
\text { Hema. } \\
\text { Anal. } \\
\\
\text { Anal. } \\
\text { Anal. } \\
\text { Anal. } \\
\text { Anal. } \\
\text { Anal. } \\
\text { Sani. } \\
\text { Anal. }\end{array}$ & $\begin{array}{l}\text { Mont. } \\
\text { Mont. } \\
\text { Mont. } \\
\text { Mont. } \\
\text { Mont. } \\
\text { Mont. } \\
\text { Mont. } \\
\text { Mont. } \\
\text { Mont. } \\
\text { Mont. } \\
\text { Mont. } \\
\text { Mont. } \\
\text { Mont. } \\
\text { Mont. } \\
\text { Mont. }\end{array}$ & $\begin{array}{l}\text { Plag. } \\
\text { Anal. } \\
\text { Sani. } \\
\text { Feld. } \\
\text { Hema. } \\
\text { Hema. } \\
\text { Sani. } \\
\text { Sani. } \\
\text { Sani. } \\
\text { Sani. } \\
\text { Sani. } \\
\text { Sani. } \\
\text { Anal. } \\
\text { Hema. }\end{array}$ & Anal. \\
\hline 927.5 & $\begin{array}{l}\text { Basalts with } \\
\text { siltstones }\end{array}$ & 它吾 & Plag. & Mont. & Hema. & Plag. & Quar. & Hema. & Mont. & Plag. & Mica \\
\hline
\end{tabular}

TABLE 6

Summary of X-Ray Mineralogy Samples, Sample Depths, Lithology, Age, and X-Ray Diffraction Results, Hole 317B

\begin{tabular}{|c|c|c|c|c|c|c|c|c|c|c|c|}
\hline \multirow{2}{*}{$\begin{array}{l}\text { Sample } \\
\text { Depth Below } \\
\text { Sea Floor } \\
\text { (m) }\end{array}$} & \multirow[b]{2}{*}{ Lithology } & \multirow[b]{2}{*}{ Age } & \multicolumn{3}{|c|}{$\begin{array}{c}\text { Bulk Sample } \\
\text { Major Constituent }\end{array}$} & \multicolumn{3}{|c|}{$\begin{array}{l}2-20 \mu \mathrm{m} \text { Fraction } \\
\text { Major Constituent }\end{array}$} & \multicolumn{3}{|c|}{$\begin{array}{c}<2 \mu \mathrm{m} \text { Fraction } \\
\text { Major Constituent }\end{array}$} \\
\hline & & & 1 & 2 & 3 & 1 & 2 & 3 & 1 & 2 & 3 \\
\hline $\begin{array}{r}0.3 \\
27.4 \\
65.7 \\
102.2 \\
141.2 \\
179.5 \\
220.7 \\
227.1 \\
266.8 \\
\end{array}$ & $\begin{array}{c}\text { Unit 1: } \\
\text { Nanno-foram } \\
\text { oozes and } \\
\text { chalk }\end{array}$ & 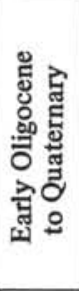 & $\begin{array}{l}\text { Calc. } \\
\text { Calc. } \\
\text { Calc. } \\
\text { Calc. } \\
\text { Calc. } \\
\text { Calc. } \\
\text { Calc. } \\
\text { Calc. } \\
\text { Calc. }\end{array}$ & & & $\begin{array}{l}\text { Bari. } \\
\text { Bari. } \\
\text { Bari. } \\
\text { Bari. } \\
\text { K-Fe. } \\
\text { Bari. } \\
\text { Psil. } \\
\text { Plag. } \\
\text { Clin. }\end{array}$ & $\begin{array}{l}\text { Plag. } \\
\text { K-Fe. } \\
\text { K-Fe. } \\
\text { Plag. } \\
\text { Bari. } \\
\text { K-Fe. } \\
\\
\text { Quar. } \\
\text { K-Fe. }\end{array}$ & $\begin{array}{l}\text { K-Fe. } \\
\text { Plag. } \\
\text { Plag. } \\
\text { K-Fe. } \\
\text { Mont. } \\
\text { Plag. } \\
\\
\text { Mica } \\
\text { Mica }\end{array}$ & $\begin{array}{l}\text { Mont. } \\
\text { Mont. } \\
\text { Mont. } \\
\text { Bari. } \\
\text { Mont. } \\
\text { Mont. } \\
\text { Psil. } \\
\text { Mont. } \\
\text { Mont. }\end{array}$ & $\begin{array}{l}\text { Plag. } \\
\text { K-Fe. } \\
\text { Apat. } \\
\text { K-Fe. } \\
\text { K-Fe. } \\
\text { Bari. } \\
\text { Gyps. } \\
\text { Mica } \\
\text { Mica }\end{array}$ & $\begin{array}{l}\text { Bari. } \\
\text { Bari. } \\
\text { Mont. } \\
\text { Mica } \\
\text { Mica } \\
\\
\text { Quar. } \\
\text { Quar. }\end{array}$ \\
\hline $\begin{array}{l}312.4 \\
352.0 \\
389.4\end{array}$ & $\begin{array}{l}\text { Unit 2: } \\
\text { As in } \\
\text { Hole } 317 \mathrm{~A}\end{array}$ & 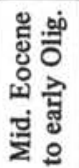 & $\begin{array}{l}\text { Calc. } \\
\text { Calc. } \\
\text { Calc. }\end{array}$ & & & $\begin{array}{l}\text { In } \\
\text { Clin. } \\
\text { Clin. }\end{array}$ & $\begin{array}{l}\text { ffic. res } \\
\text { Bari. } \\
\text { K-Fe. }\end{array}$ & $\begin{array}{l}\text { lue } \\
\text { K-Fe. } \\
\text { Bari. }\end{array}$ & $\begin{array}{l}\text { Mont. } \\
\text { Mont. } \\
\text { Cris. }\end{array}$ & $\begin{array}{l}\text { Mica } \\
\text { Mica } \\
\text { Mont. }\end{array}$ & $\begin{array}{l}\text { Chlo. } \\
\text { K-Fe. } \\
\text { Mica }\end{array}$ \\
\hline
\end{tabular}


TABLE 7

Summary of X-Ray Mineralogy Samples, Sample Depths, Lithology, Age, and X-Ray Diffraction Results, Site 318

\begin{tabular}{|c|c|c|c|c|c|c|c|c|c|c|c|}
\hline \multirow{2}{*}{$\begin{array}{l}\text { Sample } \\
\text { Depth Below } \\
\text { Sea Floor } \\
\text { (m) }\end{array}$} & \multirow[b]{2}{*}{ Lithology } & \multirow[b]{2}{*}{ Age } & \multicolumn{3}{|c|}{$\begin{array}{c}\text { Bulk Sample } \\
\text { Major Constituent }\end{array}$} & \multicolumn{3}{|c|}{$\begin{array}{c}2-20 \mu \mathrm{m} \text { Fraction } \\
\text { Major Constituent }\end{array}$} & \multicolumn{3}{|c|}{$\begin{array}{c}<2 \mu \mathrm{m} \text { Fraction } \\
\text { Major Constituent }\end{array}$} \\
\hline & & & 1 & 2 & 3 & 1 & 2 & 3 & 1 & 2 & 3 \\
\hline 1.3 & Unit $1:^{\mathrm{a}}$ & a & Calc. & Arag. & & Dolo. & Phil. & Plag. & Mont. & $\mathrm{K}-\mathrm{Fe}$. & Plag. \\
\hline $\begin{array}{r}93.7 \\
122.5 \\
179.7\end{array}$ & $\begin{array}{c}\text { Unit 2: } \\
\text { Foram-nanno } \\
\text { ooze }\end{array}$ & 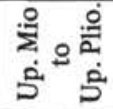 & $\begin{array}{l}\text { Calc. } \\
\text { Calc. } \\
\text { Calc. }\end{array}$ & & & $\begin{array}{r}\text { In } \\
\text { In } \\
\text { Bari. }\end{array}$ & $\begin{array}{l}\text { fffic. re } \\
\text { affic. re } \\
\text { Plag. }\end{array}$ & $\begin{array}{l}\text { due } \\
\text { due } \\
\mathrm{K}-\mathrm{Fe} \text {. }\end{array}$ & $\begin{array}{l}\text { Mont. } \\
\text { Ins } \\
\text { Mont. }\end{array}$ & $\begin{array}{l}\text { Plag. } \\
\text { ffic. res } \\
\text { Bari. }\end{array}$ & K-Fe. \\
\hline $\begin{array}{l}266.5 \\
350.8\end{array}$ & $\begin{array}{c}\text { Unit 3: } \\
\text { Foram-nanno } \\
\text { chalk }\end{array}$ & 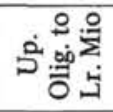 & $\begin{array}{l}\text { Calc. } \\
\text { Calc. }\end{array}$ & & & $\begin{array}{l}\text { Bari. } \\
\text { Quar. }\end{array}$ & $\begin{array}{l}\text { K-Fe. } \\
\text { Plag. }\end{array}$ & $\begin{array}{l}\text { Mica } \\
\text { Mica }\end{array}$ & $\begin{array}{l}\text { Mont. } \\
\text { Mont. }\end{array}$ & $\begin{array}{l}\text { K-Fe. } \\
\text { K-Fe. }\end{array}$ & $\begin{array}{l}\text { Bari. } \\
\text { Quar. }\end{array}$ \\
\hline $\begin{array}{l}407.5 \\
492.7\end{array}$ & $\begin{array}{c}\text { Unit 4: } \\
\text { Nanno-foram } \\
\text { limestone }\end{array}$ & 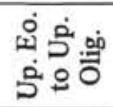 & $\begin{array}{l}\text { Calc. } \\
\text { Calc. }\end{array}$ & & & $\begin{array}{l}\text { Clin. } \\
\text { Clin. }\end{array}$ & $\begin{array}{l}\text { K-Fe. } \\
\text { K-Fe. }\end{array}$ & $\begin{array}{l}\text { Quar. } \\
\text { Hema. }\end{array}$ & $\begin{array}{l}\text { Mont. } \\
\text { Mont. }\end{array}$ & $\begin{array}{l}\text { K-Fe. } \\
\text { K-Fe. }\end{array}$ & $\begin{array}{l}\text { Plag. } \\
\text { Clin. }\end{array}$ \\
\hline $\begin{array}{l}578.5 \\
581.1 \\
615.8 \\
630.7 \\
665.6 \\
703.8 \\
734.5\end{array}$ & $\begin{array}{l}\text { Unit 5: } \\
\text { Limestone, } \\
\text { siltstone, } \\
\text { and graded } \\
\text { sandstone }\end{array}$ & 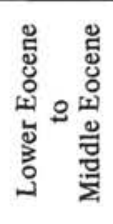 & $\begin{array}{l}\text { Calc. } \\
\text { Calc. } \\
\text { Mont. } \\
\text { Mont. } \\
\text { Mont. } \\
\text { K-Fe. } \\
\text { Mont. }\end{array}$ & $\begin{array}{l}\text { Clin. } \\
\text { Calc. } \\
\text { Calc. } \\
\text { Plag. } \\
\text { Clin. } \\
\text { Calc. }\end{array}$ & $\begin{array}{l}\text { K-Fe. } \\
\text { K-Fe. } \\
\text { K-Fe. } \\
\text { Mont. } \\
\text { Clin. }\end{array}$ & $\begin{array}{l}\text { Clin. } \\
\text { Clin. } \\
\text { Clin. } \\
\text { Mont. } \\
\text { Mont. } \\
\text { Clin. } \\
\text { K-Fe. }\end{array}$ & $\begin{array}{l}\text { K-Fe. } \\
\text { K-Fe. } \\
\text { Mont. } \\
\text { K-Fe. } \\
\text { K-Fe. } \\
\text { K-Fe. } \\
\text { Plag. }\end{array}$ & $\begin{array}{l}\text { Mont. } \\
\text { Plag. } \\
\text { K-Fe. } \\
\text { Plag. } \\
\text { Clin. } \\
\text { Mont. } \\
\text { Mont. }\end{array}$ & $\begin{array}{l}\text { Mont. } \\
\text { Mont. } \\
\text { Mont. } \\
\text { Mont. } \\
\text { Mont. } \\
\text { Mont. } \\
\text { Mont. }\end{array}$ & $\begin{array}{l}\text { K-Fe. } \\
\text { K-Fe. } \\
\text { K-Fe. } \\
\text { K-Fe. } \\
\text { K-Fe. }\end{array}$ & $\begin{array}{l}\text { Plag. } \\
\text { Plag.. } \\
\text { Plag. } \\
\text { Plag. } \\
\text { Plag. }\end{array}$ \\
\hline
\end{tabular}

${ }^{\mathrm{a}}$ Nannofossil-foraminiferal ooze with graded shell beds; Quaternary.

TABLE 8

Results of X-Ray Diffraction Analysis, Site 314

\begin{tabular}{|c|c|c|c|c|c|c|c|c|c|c|c|c|c|c|c|c|c|c|c|}
\hline $\begin{array}{l}\text { Sample } \\
\text { Depth Below } \\
\text { Sea Floor }\end{array}$ & Amor. & Dolo. & Quar. & K-Fe. & Cris. & Plag. & Mica & Chlo. & Goet. & Paly. & Mont. & Trid. & Clin. & Phil. & Gyps. & Sepi. & Magn. & Cupr. ${ }^{a}$ & Anat. \\
\hline \multicolumn{20}{|l|}{ Bulk Sample } \\
\hline $\begin{array}{l}0.0 \\
0 \text { to } 45^{b} \\
0 \text { to } 45^{b}\end{array}$ & $\begin{array}{l}75.9 \\
59.8 \\
52.2\end{array}$ & $\begin{array}{l}- \\
-\end{array}$ & $\begin{array}{r}3.8 \\
9.3 \\
13.2\end{array}$ & $\begin{array}{l}2.0 \\
- \\
-\end{array}$ & & $\begin{array}{l}- \\
9.3 \\
7.2\end{array}$ & $\begin{array}{r}1.4 \\
13.9 \\
18.3\end{array}$ & & $\begin{array}{r}16.4 \\
4.6 \\
2.6\end{array}$ & $\overline{-}$ & $\begin{array}{r}12.0 \\
6.4 \\
5.6\end{array}$ & & $\begin{array}{r}31.5 \\
9.3 \\
2.6\end{array}$ & $\begin{array}{l}3 \overline{9.4} \\
45.0\end{array}$ & & $\begin{array}{l}- \\
4.8 \\
2.7\end{array}$ & $\begin{array}{r}18.2 \\
3.0 \\
0.3\end{array}$ & $\begin{array}{c}14.7 \\
- \\
-\end{array}$ & \\
\hline \multicolumn{20}{|c|}{$2-20, \mu \mathrm{m}$ Fraction } \\
\hline $\begin{array}{l}0.0 \\
0 \text { to } 45 \\
0 \text { to } 45\end{array}$ & $\begin{array}{l}52.8 \\
24.5 \\
25.3\end{array}$ & & $\begin{array}{r}5.2 \\
11.7 \\
18.7\end{array}$ & $\begin{array}{l}4.0 \\
- \\
-\end{array}$ & & $\begin{array}{l}\overline{8.1} \\
9.9\end{array}$ & $\begin{array}{r}3.9 \\
14.2 \\
21.5\end{array}$ & $\begin{array}{l}- \\
1.6 \\
2.4\end{array}$ & $\begin{array}{r}10.8 \\
7.0 \\
-\end{array}$ & $\begin{array}{l}- \\
1.9\end{array}$ & $\begin{array}{l}\overline{2} .2 \\
-\end{array}$ & & $\begin{array}{r}50.7 \\
13.1 \\
3.9\end{array}$ & $\begin{array}{l}3 \overline{7} .7 \\
38.5\end{array}$ & & $\begin{array}{l}-\overline{1.0} \\
2.6\end{array}$ & $\begin{array}{r}18.2 \\
3.4 \\
-\end{array}$ & $\begin{array}{l}7.2 \\
- \\
-\end{array}$ & $\overline{0}$ \\
\hline \multicolumn{20}{|c|}{$<2 \mu$ m Fraction } \\
\hline $\begin{array}{l}0.0 \\
0 \text { to } 45 \\
0 \text { to } 45\end{array}$ & $\begin{array}{l}73.3 \\
71.0 \\
77.3\end{array}$ & & $\begin{array}{r}3.2 \\
9.7 \\
14.3\end{array}$ & & $\begin{array}{c}16.0 \\
- \\
-\end{array}$ & $\overline{-}$ & $\begin{array}{l}\overline{-} \\
11.3 \\
20.8\end{array}$ & $\frac{-}{3.4}$ & $\begin{array}{r}15.9 \\
8.2 \\
-\end{array}$ & $\overline{7.3}$ & $\begin{array}{l}39.4 \\
31.8 \\
27.3\end{array}$ & $\begin{array}{l}4.5 \\
- \\
-\end{array}$ & $\begin{array}{l}4.0 \\
1.0 \\
-\end{array}$ & $\begin{array}{r}3 \overline{0} .0 \\
7.7\end{array}$ & $\begin{array}{l}- \\
\overline{0.2}\end{array}$ & $\begin{array}{l}-\overline{4} \\
4.9\end{array}$ & $\begin{array}{c}12.2 \\
3.7 \\
-\end{array}$ & $\begin{array}{l}4.7 \\
- \\
-\end{array}$ & \\
\hline
\end{tabular}

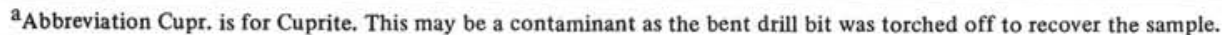

${ }^{\mathrm{b}}$ Aggregated mud samples of uncertain depth. 
TABLE 9

Results of X-Ray Diffraction Analysis, Hole 315

\begin{tabular}{|c|c|c|c|c|c|c|c|c|c|c|c|c|c|c|c|c|}
\hline $\begin{array}{c}\text { Sample } \\
\text { Depth Belou } \\
\text { Sea Floor } \\
\text { (m) }\end{array}$ & Amor. & Calc. & Quar. & K-Fe. & Plag. & Kaol. & Mica & Chlo. & Paly. & Mont. & Clin. & Phil. & Gyps. & Bari. & Amph. & Anat. \\
\hline \multicolumn{17}{|l|}{ Bulk Sample } \\
\hline 1.30 & 20.4 & 99.7 & 0.3 & & & & & & & & & & - & - & & \\
\hline 3.90 & 24.7 & 99.5 & 0.3 & & & & & & & & & & 0.2 & - & & \\
\hline 57.90 & 47.6 & 97.3 & 1.1 & & & & & & & & & & 0.3 & 1.2 & & \\
\hline 59.20 & 26.8 & 99.7 & 0.3 & & & & & & & & & & - & - & & \\
\hline 61.90 & 26.9 & 99.1 & 0.3 & & & & & & & & & & - & 0.6 & & \\
\hline 65.50 & 19.0 & 100.0 & - & & & & & & & & & & - & - & & \\
\hline \multicolumn{17}{|c|}{ 2-20 $\mu$ m Fraction } \\
\hline 1.30 & 87.6 & & 27.6 & 7.7 & 16.9 & - & 25.8 & 5.9 & - & 2.3 & - & - & & 13.9 & - & - \\
\hline 3.90 & 86.7 & & 28.2 & - & 16.7 & - & 18.7 & 3.5 & - & - & - & 18.1 & & 14.3 & - & 0.5 \\
\hline 57.90 & 89.8 & & 10.4 & 3.4 & 8.1 & - & 13.9 & 6.9 & - & 7.0 & - & 3.9 & & 46.4 & - & - \\
\hline 59.20 & 86.7 & & 15.8 & 16.3 & 10.6 & - & 13.4 & 2.7 & - & 2.5 & - & - & & 37.1 & 1.5 & - \\
\hline 61.90 & 83.8 & & 7.8 & 9.3 & 9.3 & 1.3 & 10.3 & 1.9 & 5.7 & 8.2 & 0.5 & - & & 45.8 & - & - \\
\hline 65.50 & 91.6 & & 13.1 & 24.3 & 15.8 & - & 15.6 & 9.1 & - & - & - & - & & 22.2 & - & - \\
\hline \multicolumn{17}{|c|}{$<2 \mu \mathrm{m}$ Fraction } \\
\hline 1.30 & 86.3 & & 18.1 & 2.6 & 7.7 & 4.2 & 21.8 & 2.9 & 4.7 & 32.6 & & - & 1.0 & 4.5 & - & \\
\hline 3.90 & 88.9 & & 16.7 & - & 9.1 & - & 17.7 & 2.2 & - & 35.3 & & 11.7 & 4.1 & 2.1 & 1.2 & \\
\hline 57.90 & 84.2 & & 7.8 & - & 5.6 & - & 8.5 & - & 7.2 & 40.2 & & 24.1 & 1.7 & 4.9 & - & \\
\hline 59.20 & 86.0 & & 10.3 & 7.4 & 12.0 & 1.8 & 7.7 & 2.1 & - & 51.6 & & - & 1.7 & 5.3 & - & \\
\hline 61.90 & 84.4 & & 8.0 & 2.3 & 6.5 & - & 8.3 & 1.7 & 3.2 & 51.9 & & 11.8 & - & 6.4 & - & \\
\hline 65.50 & 85.5 & & 8.1 & 0.7 & 4.9 & - & 8.4 & 4.5 & - & 54.8 & & 13.1 & 1.6 & 3.9 & - & \\
\hline
\end{tabular}


TABLE 10

Results of X-Ray Diffraction Analysis, Hole 315A

\begin{tabular}{|c|c|c|c|c|c|c|c|c|c|c|c|c|c|c|c|c|c|c|c|c|c|c|c|c|c|}
\hline $\begin{array}{c}\text { Sample } \\
\text { Depth Below } \\
\text { Sea Floor } \\
\text { (m) }\end{array}$ & Amor. & Calc. & Dolo. & Arag. & Feld. $^{a}$ & Quar. & Cris. & K-Fe. & Plag. & Kaol. & Mica & Chlo. & Goet. & Paly. & Mont. & Trid. & Clin. & Phil. & Pyri. & Hema. & Gyps. & Bari. & Amph. & Gibb. & Magn. \\
\hline \multicolumn{26}{|l|}{ Bulk Samples } \\
\hline 76.6 & 20.5 & 100.0 & - & - & & - & - & - & - & & - & & & & - & - & - & & & - & - & - & & & - \\
\hline 82.90 & 17.3 & 99.7 & - & - & & 0.3 & - & - & - & & - & & & & - & - & - & & & - & - & - & & & - \\
\hline 124.90 & 19.2 & 100.0 & - & - & & - & - & - & - & & - & & & & - & - & - & & & - & - & - & & & - \\
\hline 142.60 & 19.7 & 99.4 & - & - & & 0.2 & - & - & - & & - & & & & - & - & - & & & - & - & 0.4 & & & - \\
\hline 148.90 & 20.8 & 99.2 & - & - & & 0.2 & - & - & - & & - & & & & - & - & - & & & - & - & 0.6 & & & $\therefore$ \\
\hline 260.00 & 18.7 & 100.0 & - & - & & - & - & - & - & & - & & & & - & - & - & & & - & - & - & & & - \\
\hline 372.10 & 18.7 & 98.6 & - & - & & 0.1 & - & - & - & & - & & & & - & - & - & & & 0.9 & - & 0.3 & & & - \\
\hline 465.90 & 17.1 & 100.0 & - & - & & - & - & - & - & & - & & & & - & - & - & & & - & - & - & & & - \\
\hline 513.50 & 12.1 & 100.0 & - & - & & - & - & - & - & & - & & & & - & - & - & & & - & - & - & & & - \\
\hline 591.00 & 10.2 & 100.0 & - & - & & - & - & - & - & & - & & & & - & - & - & & & - & - & - & & & - \\
\hline 703.60 & 18.2 & 100.0 & - & - & & - & - & - & - & & - & & & & - & - & - & & & - & - & - & & & - \\
\hline 731.30 & 10.0 & 98.4 & - & - & & 0.2 & - & - & - & & - & & & & - & - & 0.5 & & & - & - & 0.9 & & & - \\
\hline 739.30 & 88.3 & 61.7 & - & - & & 3.9 & - & - & - & & 2.3 & & & & 14.4 & - & - & & & - & - & 17.7 & & & - \\
\hline 741.40 & 13.7 & 26.7 & - & - & & 48.1 & 22.3 & - & - & & - & & & & - & 2.9 & - & & & - & - & - & & & - \\
\hline 779.20 & 74.8 & 14.6 & - & - & & 23.6 & - & 8.7 & 6.6 & & 12.4 & & & & 14.9 & - & 13.8 & & & - & 0.5 & 5.1 & & & - \\
\hline 779.60 & 73.3 & - & - & - & & 25.9 & - & 5.0 & 6.1 & & 22.8 & & & & 16.9 & - & 21.1 & & & - & 0.4 & 1.8 & & & - \\
\hline 788.50 & 66.0 & - & - & - & & 26.6 & 12.1 & 3.8 & 3.2 & & 16.0 & & & & 14.0 & - & 24.3 & & & - & - & - & & & - \\
\hline 797.10 & 48.1 & - & - & - & & 28.8 & - & 7.2 & 4.7 & & 17.0 & & & & 11.3 & - & 27.0 & & & - & 1.5 & 2.4 & & & - \\
\hline 799.20 & 34.2 & 88.3 & - & - & & 4.6 & - & 0.6 & 0.4 & & 3.1 & & & & 1.5 & - & 1.5 & & & - & - & - & & & - \\
\hline 808.60 & 44.7 & 76.6 & - & - & & 8.7 & - & 1.2 & 0.8 & & 5.7 & & & & 2.9 & - & 4.1 & & & - & - & - & & & - \\
\hline 875.90 & 32.8 & 77.2 & - & - & & 8.9 & - & 1.2 & 0.7 & & 4.0 & & & & 7.4 & - & 0.6 & & & - & - & - & & & - \\
\hline 893.40 & 47.2 & 50.4 & - & - & & 36.8 & - & 1.6 & 1.6 & & 2.2 & & & & 3.4 & - & 4.2 & & & - & - & - & & & - \\
\hline 911.30 & 56.4 & 17.1 & - & - & & 48.2 & - & 6.4 & 4.2 & & 12.3 & & & & 6.9 & - & 2.9 & & & 1.9 & - & - & & & - \\
\hline 932.70 & 45.7 & - & - & - & & 0.6 & - & 43.9 & 4.1 & & 0.9 & & & & 32.4 & - & 16.9 & & & 1.2 & - & - & & & - \\
\hline 952.80 & 51.8 & - & - & - & & 75.8 & - & - & - & & 14.8 & & & & 9.4 & - & - & & & - & - & - & & & - \\
\hline 952.90 & 44.5 & 93.6 & - & - & & - & - & - & - & & 2.3 & & & & 1.2 & - & - & & & 2.9 & - & - & & & - \\
\hline 970.90 & 45.3 & 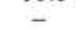 & - & 8.0 & & 19.9 & - & 2.9 & 1.4 & & 36.0 & & & & 31.3 & - & 0.4 & & & - & 0.1 & - & & & - \\
\hline 990.00 & 70.8 & - & - & - & & 53.9 & - & 4.1 & 1.9 & & 2.5 & & & & 24.2 & - & - & & & 13.4 & - & - & & & - \\
\hline \multicolumn{26}{|c|}{$2.20 \mu \mathrm{m}$ Fraction } \\
\hline 82.90 & 86.1 & & & & - & 7.9 & - & - & 10.1 & & 9.1 & - & - & - & - & - & - & - & - & - & - & 11.2 & 61.7 & - & - \\
\hline 124.90 & 96.1 & & & & - & 5.7 & - & 19.6 & 13.4 & & 9.6 & - & - & - & - & - & - & - & - & - & - & 51.7 & - & - & - \\
\hline 142.60 & 90.8 & & & & - & 2.4 & - & 10.4 & 6.8 & & - & - & - & - & - & - & - & - & 4.1 & - & - & 76.3 & - & - & - \\
\hline 148.90 & 97.4 & & & & - & 8.4 & - & - & - & & 16.2 & 20.0 & - & - & - & - & - & - & 13.6 & - & - & 41.8 & - & - & - \\
\hline 260.00 & 76.9 & & & & - & 2.1 & - & - & 40.4 & & 3.5 & - & - & - & 9.0 & - & - & - & 43.2 & - & - & 1.8 & - & - & - \\
\hline 372.10 & 95.5 & & & & - & 6.1 & - & 15.7 & 13.6 & & - & - & - & - & - & - & - & - & 8.7 & - & - & 55.9 & - & - & - \\
\hline 465.90 & 94.6 & & & & - & 4.4 & - & 14.3 & 12.4 & & - & - & - & - & - & - & - & - & - & - & - & 68.8 & - & - & - \\
\hline 513.50 & 91.5 & & & & - & -7 & - & 22.9 & 4.7 & & - & - & - & - & - & - & - & - & - & - & - & 72.4 & - & - & - \\
\hline 591.00 & 95.2 & & & & 15.2 & 5.9 & - & - & - & & - & - & - & - & - & - & - & - & - & - & - & 78.9 & - & - & - \\
\hline 703.60 & 88.5 & & & & - & 1.0 & - & 13.8 & 13.8 & & 6.1 & 2.2 & - & - & 6.7 & - & - & 7.6 & - & - & 0.1 & 48.7 & - & - & - \\
\hline 731.30 & 37.6 & & & & - & 1.9 & - & 8.2 & 5.4 & & 3.4 & - & - & - & 5.7 & - & 57.5 & - & - & - & - & 17.8 & - & - & - \\
\hline 739.30 & 90.3 & & & & - & 6.9 & - & 13.2 & 10.3 & & - & - & - & - & 18.5 & - & - & - & - & - & - & 51.0 & - & - & - \\
\hline 741.40 & 13.5 & & & & _- & 35.6 & 56.7 & - & - & & - & - & - & - & - & 7.6 & - & - & - & - & - & - & - & - & - \\
\hline 779.20 & 59.6 & & & & - & 28.6 & - & 8.7 & 9.5 & & 12.4 & - & - & - & 8.1 & - & 24.3 & - & - & - & - & 8.4 & - & - & - \\
\hline 779.60 & 48.7 & & & & - & 25.1 & - & 11.9 & 8.3 & & 13.3 & - & - & - & 5.1 & - & 36.3 & - & - & - & - & - & - & - & - \\
\hline 788.50 & 47.3 & & & & - & 26.7 & 15.5 & 5.1 & 3.6 & & 4.4 & - & 12.5 & - & 6.3 & - & 25.7 & - & - & - & - & - & - & - & - \\
\hline 797.10 & 37.3 & & & & $=$ & 25.1 & - & 9.2 & 6.0 & & 11.2 & 0.7 & - & $=$ & 4.0 & 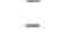 & 43.8 & 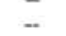 & - & - & - & - & - & - & - \\
\hline 799.20 & 53.2 & & & & - & 40.8 & - & 9.5 & 5.4 & & 18.5 & - & - & - & 6.1 & - & 19.7 & - & - & - & - & - & - & - & - \\
\hline 808.60 & 36.8 & & & & - & 28.6 & - & 12.7 & 6.6 & & 7.4 & - & - & - & - & - & 44.7 & - & - & - & - & - & - & - & - \\
\hline 818.70 & 47.6 & & & & - & 23.6 & - & 17.0 & 5.9 & & 6.8 & - & - & - & 4.3 & - & 42.4 & - & - & - & - & - & - & - & - \\
\hline 826.90 & 49.2 & & & & - & 36.5 & - & 19.7 & 2.3 & & 23.4 & - & - & - & 5.0 & - & 13.2 & - & - & - & - & - & - & - & - \\
\hline 836.80 & 44.6 & & & & - & 25.6 & - & 31.0 & 10.2 & & 9.9 & 0.7 & - & $=$ & 0.7 & $=$ & 14.3 & - & - & 7.4 & - & - & - & 0.3 & - \\
\hline 837.00 & 41.1 & & & & - & 9.7 & - & 9.8 & 6.4 & & 5.7 & - & - & - & 5.5 & - & 55.6 & - & - & - & - & - & - & - & 7.3 \\
\hline 850.50 & 34.5 & & & & - & - & -. & 7.1 & 37.3 & & 1.2 & - & - & 0.8 & 47.6 & - & 2.3 & - & - & - & - & - & - & - & 3.8 \\
\hline 856.00 & 23.8 & & & & - & - & - & 66.5 & 11.7 & & 1.0 & - & - & - & 12.2 & - & 3.9 & - & - & - & - & - & - & - & 4.7 \\
\hline 858.30 & 38.2 & & & & - & 1.1 & - & 36.8 & 5.8 & & 1.6 & - & - & - & 49.0 & - & 5.6 & - & - & - & - & - & - & - & - \\
\hline 875.90 & 48.0 & & & & - & 53.2 & - & 10.1 & 3.8 & & 15.3 & - & - & - & 16.7 & - & - & - & - & - & - & - & - & - & 0.9 \\
\hline 893.40 & 44.6 & & & & - & 69.5 & - & 6.4 & 3.9 & & 5.9 & _ & - & - & 3.9 & - & 9.4 & - & - & - & - & - & - & - & 1.0 \\
\hline
\end{tabular}




\begin{tabular}{|c|c|c|c|c|c|c|c|c|c|c|c|c|c|c|c|c|c|c|c|c|c|c|}
\hline 911.30 & 41.6 & - & 56.8 & - & 11.0 & 7.7 & & 10.0 & - & - & - & 3.6 & - & 7.9 & - & - & 1.8 & - & - & - & - & 1.3 \\
\hline 932.70 & 50.6 & - & 1.0 & - & 58.1 & 11.1 & & 1.0 & - & - & - & 23.8 & - & 3.0 & - & - & 2.1 & - & - & - & - & - \\
\hline 952.80 & 41.5 & - & 90.0 & - & - & - & & 6.8 & - & - & - & 3.2 & - & - & - & - & - & - & - & - & - & - \\
\hline 952.90 & 37.1 & - & 92.2 & - & - & - & & 4.7 & - & - & - & - & - & - & - & - & 3.1 & - & - & - & - & - \\
\hline 970.90 & 49.2 & - & 70.9 & - & 1.7 & 2.8 & & 17.8 & - & - & - & 6.7 & - & - & - & - & - & - & - & - & - & - \\
\hline 990.00 & 59.6 & - & 43.2 & - & 9.1 & 3.3 & & 11.1 & 1.8 & - & - & 15.3 & - & - & - & - & 16.1 & - & - & - & - & - \\
\hline \multicolumn{23}{|c|}{$<2 \mu \mathrm{m}$ Fraction } \\
\hline 76.6 & 58.2 & & 7.9 & - & 7.3 & 9.1 & - & 15.6 & - & - & - & 55.9 & - & - & - & 4.2 & - & - & - & & - & - \\
\hline 82.90 & 83.5 & & 6.1 & - & 1.2 & 3.3 & 1.4 & 9.6 & - & - & 7.4 & 56.2 & - & - & 3.4 & 2.4 & - & 1.6 & 7.5 & & - & - \\
\hline 124.90 & 89.0 & & 4.4 & - & - & - & - & 4.5 & 3.7 & - & - & 63.7 & - & - & 12.8 & - & - & 1.1 & 9.8 & & - & - \\
\hline 142.60 & 92.0 & & 48.0 & - & 5.2 & 4.5 & - & 2.4 & 2.0 & - & - & 27.6 & - & - & - & - & - & 0.5 & 9.9 & & - & - \\
\hline 148.90 & 87.3 & & 3.5 & - & 7.5 & 6.1 & - & 7.9 & 1.3 & - & - & 62.8 & - & - & - & - & - & - & 10.9 & & - & - \\
\hline 260.00 & 80.9 & & 4.3 & - & 4.9 & 3.9 & - & 3.9 & 1.9 & - & 6.0 & 68.9 & - & - & - & 2.9 & - & 0.6 & -2.6 & & - & - \\
\hline 372.10 & 94.4 & & 11.6 & - & 7.7 & 10.0 & - & 8.6 & 3.5 & - & - & 31.0 & - & - & - & 3.9 & - & 1.6 & 22.1 & & - & - \\
\hline 465.90 & 92.8 & & 11.4 & - & 2.0 & 6.8 & - & 6.6 & 3.0 & - & - & 27.7 & - & - & 14.5 & - & - & 0.9 & 26.4 & & 0.6 & - \\
\hline 513.50 & 82.7 & & 6.4 & - & 5.1 & 3.7 & - & 2.6 & 3.5 & - & - & 52.7 & - & - & 6.0 & - & - & 0.9 & 19.1 & & - & - \\
\hline 591.00 & 89.3 & & 5.9 & - & 11.3 & 6.1 & - & 4.8 & 2.8 & - & - & 51.5 & - & - & - & - & - & - & 17.6 & & - & - \\
\hline 703.60 & 78.2 & & 5.3 & - & 9.7 & 7.6 & - & - & 2.6 & - & - & 53.4 & - & - & - & - & - & - & 21.4 & & - & - \\
\hline 731.30 & 63.0 & & 1.4 & - & 10.3 & 8.1 & - & - & - & - & - & 39.8 & - & 15.4 & - & - & - & 0.2 & 24.7 & & - & - \\
\hline 739.30 & 84.3 & & 8.8 & - & 9.8 & 9.8 & - & - & - & - & - & 63.1 & - & - & - & - & - & 0.7 & 7.8 & & - & - \\
\hline 741.40 & 20.4 & & 11.1 & 78.4 & - & - & - & - & - & - & - & 2.7 & 7.8 & - & - & - & - & - & - & & - & - \\
\hline 779.20 & 78.2 & & 31.2 & - & 9.0 & 6.8 & - & 14.6 & - & - & - & 27.4 & - & 7.2 & - & - & - & - & 3.8 & & - & - \\
\hline 779.60 & 74.4 & & 31.1 & - & 8.6 & 6.1 & - & 19.7 & - & - & - & 21.2 & - & 13.4 & - & - & - & - & - & & - & - \\
\hline 788.50 & 70.2 & & 30.0 & 14.2 & - & 1.1 & - & 8.9 & - & 9.0 & - & 35.7 & - & 0.8 & - & - & - & 0.3 & - & & - & - \\
\hline 797.10 & 70.9 & & 33.2 & - & 7.0 & 3.2 & - & 19.1 & - & - & - & 25.9 & - & 8.6 & - & - & - & 0.1 & 2.9 & & - & - \\
\hline 799.20 & 73.1 & & 32.5 & - & 10.4 & 1.9 & - & 17.2 & - & - & - & 34.9 & - & 2.8 & - & - & - & 0.2 & - & & - & - \\
\hline 808.60 & 71.6 & & 35.9 & - & 5.6 & 1.5 & - & 26.4 & - & - & - & 27.7 & - & 2.9 & - & - & - & - & - & & - & - \\
\hline 818.70 & 67.7 & & 32.7 & - & 5.1 & 3.1 & - & 12.1 & - & - & - & 44.9 & - & 1.8 & - & - & - & 0.3 & - & & - & - \\
\hline 826.90 & 72.6 & & 38.4 & - & 4.9 & 4.5 & - & 28.6 & - & - & - & 22.7 & - & 0.8 & - & - & - & - & - & & - & - \\
\hline 836.80 & 58.1 & & 45.4 & - & 3.5 & 1.4 & - & 4.2 & - & - & - & 39.5 & - & 4.0 & - & - & 1.7 & 0.2 & - & & - & - \\
\hline 837.00 & 57.5 & & 13.3 & - & 2.6 & 2.8 & - & 5.8 & - & - & - & 74.0 & - & 1.6 & - & - & - & - & - & & - & - \\
\hline 850.50 & 92.0 & & - & - & - & - & - & - & - & - & - & 100.0 & - & - & - & - & - & - & - & & - & - \\
\hline 856.00 & 57.3 & & 0.4 & - & 55.3 & 6.7 & - & 4.8 & - & - & - & 28.0 & - & 1.2 & - & - & - & - & - & & - & 3.5 \\
\hline 858.30 & 60.5 & & 1.9 & - & 17.5 & 3.2 & - & - & - & - & - & 77.4 & - & - & - & - & - & - & - & & - & - \\
\hline 875.90 & 58.2 & & 58.2 & - & 5.1 & 1.5 & - & 13.9 & - & - & - & 21.3 & - & - & - & - & - & - & - & & - & - \\
\hline 893.40 & 54.4 & & 75.3 & - & 1.7 & 1.1 & - & 1.7 & - & - & - & 19.2 & - & 0.6 & - & - & - & - & - & & - & 0.5 \\
\hline 911.30 & 57.0 & & 63.6 & - & 3.6 & 2.4 & - & 9.6 & - & - & - & 19.3 & - & 1.5 & - & - & - & - & - & & - & - \\
\hline 932.70 & 84.3 & & - & - & - & - & - & - & - & - & - & 100.0 & - & - & - & - & - & - & - & & - & - \\
\hline 952.80 & 2.8 & & - & 11.2 & - & - & - & 27.6 & - & - & - & 45.1 & 16.1 & - & - & - & - & - & - & & - & - \\
\hline 952.90 & 54.9 & & 75.7 & - & - & - & - & 8.9 & - & - & - & 11.5 & - & - & - & - & 3.9 & - & - & & - & - \\
\hline 970.00 & 37.6 & & 24.2 & - & - & 0.8 & - & 51.6 & - & - & - & 23.4 & - & - & - & - & - & - & - & & - & - \\
\hline 990.00 & 60.8 & & 36.8 & - & 1.8 & 2.0 & - & 2.8 & - & - & - & 45.0 & - & - & - & - & 11.5 & - & - & & - & - \\
\hline
\end{tabular}


TABLE 11

Results of X-Ray Diffraction Analysis, Site 316

\begin{tabular}{|c|c|c|c|c|c|c|c|c|c|c|c|c|c|c|c|c|c|c|c|c|c|c|c|c|c|c|}
\hline $\begin{array}{c}\text { Sample Depth } \\
\text { Below Sea } \\
\text { Floor }(\mathrm{m})\end{array}$ & Amor. & Calc. & Dolo. & Feld. $^{a}$ & Quar. & Cris. & $\mathrm{K}-\mathrm{Fe}$. & Plag. & Kaol. & Mica & Chlo. & Goet. & Paly. & Mont. & Trid. & Clin. & Phil. & Anal. & Hema. & Pyri. & Gibb. & Gyps. & Bari. & Augi. & Magn. & $\mathrm{U}-2^{\mathrm{b}}$ \\
\hline \multicolumn{27}{|l|}{ Bulk Sample } \\
\hline 0.80 & 31.8 & 97.6 & - & - & 0.4 & - & - & - & & 1.4 & & & & - & - & - & & - & - & - & - & - & 0.6 & & - & - \\
\hline $\begin{array}{r}2.70 \\
155.30\end{array}$ & $\begin{array}{l}15.8 \\
20.8\end{array}$ & $\begin{array}{r}99.8 \\
100.0\end{array}$ & - & $\bar{z}$ & 0.2 & I & E & - & & I & & & & $\overline{-}$ & - & - & & - & - & - & - & - & - & & - & - \\
\hline 268.30 & $\begin{array}{l}20.8 \\
21.6\end{array}$ & $\begin{array}{r}100.0 \\
94.5\end{array}$ & $\overline{-}$ & $\bar{z}$ & $\overline{0.2}$ & $\overline{-}$ & $\overline{3.5}$ & $\overline{1.1}$ & & $\overline{-}$ & & & & $\overline{-}$ & $\bar{z}$ & $\overline{-}$ & & $\overline{-}$ & $\overline{-}$ & $0 \overline{7}$ & $\overline{-}$ & $\bar{z}$ & $\overline{-}$ & & - & - \\
\hline 391.80 & 29.8 & 99.1 & - & - & 0.2 & - & - & - & & - & & & & $z$ & $\overline{-}$ & $\overline{-}$ & & $\overline{-}$ & $\overline{-}$ & 0.7 & $\overline{-}$ & $\overline{-}$ & $\overline{0.7}$ & & $\overline{-}$ & $\overline{-}$ \\
\hline 448.60 & 20.5 & 100.0 & - & - & - & - & - & - & & - & & & & - & - & - & & - & - & - & - & - & - & & $\overline{-}$ & $\overline{-}$ \\
\hline 469.30 & 55.2 & $\overline{-}$ & 83.4 & - & 0.8 & - & 1.3 & 0.9 & & 3.3 & & & & 3.0 & - & 5.6 & & - & - & - & - & - & 1.7 & & - & - \\
\hline 486.50 & 22.5 & 97.4 & 0.5 & - & 0.3 & - & - & - & & - & & & & - & - & 1.8 & & - & - & - & - & - & - & & - & - \\
\hline 495.90 & -7.3 & 34.6 & - & - & 5.3 & 47.1 & - & - & & 1.0 & & & & - & 11.9 & - & & - & - & - & - & - & - & & - & - \\
\hline 515.40 & 11.5 & 98.1 & 0.3 & - & 1.7 & - & - & - & & - & & & & - & - & - & & - & - & - & - & - & - & & - & - \\
\hline 524.70 & 10.0 & 99.8 & - & - & 0.2 & - & - & - & & - & & & & - & - & - & & - & - & - & - & - & - & & - & - \\
\hline 533.60 & 13.1 & 98.9 & - & - & 0.7 & - & - & - & & - & & & & - & - & - & & - & - & - & - & - & 0.4 & & - & - \\
\hline 553.30 & 29.0 & 96.2 & - & - & 1.6 & - & 0.8 & 0.5 & & 1.0 & & & & - & - & - & & - & - & - & - & - & - & & - & - \\
\hline 562.90 & 20.3 & 98.5 & - & - & 0.8 & - & - & - & & - & & & & - & - & & & - & - & - & - & - & 0.7 & & - & - \\
\hline 571.80 & 21.4 & 96.3 & - & - & 1.0 & - & - & - & & - & & & & 0.9 & - & - & & - & - & - & - & - & 1.8 & & - & - \\
\hline 573.60 & 8.7 & 98.8 & - & - & 1.2 & - & - & - & & - & & & & - & - & - & & - & - & - & - & - & - & & - & - \\
\hline 583.10 & 20.6 & 78.4 & - & - & 19.2 & - & - & - & & - & & & & - & - & 2.4 & & - & - & - & - & - & - & & - & - \\
\hline 584.40 & 74.6 & 16.3 & - & - & 36.1 & - & 5.4 & 5.2 & & 17.5 & & & & 10.0 & - & 9.3 & & - & - & - & - & 0.2 & - & & - & - \\
\hline 587.40 & 10.6 & 95.2 & - & - & 3.2 & - & - & - & & 1.6 & & & & - & - & - & & - & - & - & - & - & - & & - & 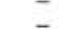 \\
\hline 609.80 & 24.8 & 81.7 & - & - & 15.1 & - & - & - & & 1.4 & & & & 1.5 & - & 0.4 & & - & - & - & - & - & - & & - & - \\
\hline 610.50 & 52.1 & 52.0 & - & - & 0.3 & - & - & - & & - & & & & 46.7 & - & 1.0 & & - & - & - & - & - & - & & - & P \\
\hline 613.60 & 9.0 & 97.1 & - & - & 2.2 & - & - & - & & - & & & & 0.7 & - & - & & - & - & - & - & - & - & & - & - \\
\hline 630.60 & 16.0 & 91.3 & - & - & 2.3 & - & - & - & & - & & & & 6.2 & - & 0.2 & & - & - & - & - & - & - & & - & - \\
\hline 638.40 & 13.4 & 96.0 & - & 0.3 & 2.7 & - & $=$ & - & & - & & & & 0.6 & - & 0.4 & & - & - & - & - & - & - & & - & - \\
\hline 641.80 & 20.4 & 80.5 & - & - & 0.1 & - & 7.4 & 4.8 & & 1.1 & & & & 4.3 & - & 0.4 & & - & 1.2 & - & - & - & - & & 0.1 & - \\
\hline 642.10 & 35.3 & 66.2 & - & - & 0.8 & - & 7.8 & 4.2 & & 1.3 & & & - & 13.6 & - & 2.2 & & - & 3.4 & - & - & - & 0.5 & & - & P \\
\hline 667.10 & 16.4 & 98.2 & - & - & 0.7 & - & - & - & & - & & & & 1.1 & - & - & & - & - & - & - & - & - & & - & - \\
\hline 668.10 & 18.8 & 95.5 & - & 0.3 & 0.7 & - & - & - & & - & & & & 3.5 & - & - & & - & - & - & - & - & - & & - & $\mathrm{P}$ \\
\hline 686.70 & 7.7 & 98.0 & - & - & 1.4 & - & - & - & & - & & & & 0.6 & - & - & & - & 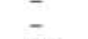 & $\overline{-}$ & $\overline{-}$ & $=$ & $\overline{-}$ & & $\overline{-}$ & - \\
\hline 704.50 & 25.9 & 78.5 & - & - & 0.6 & - & 6.2 & 1.8 & & 0.9 & & & & 11.6 & - & - & & - & 0.4 & - & - & - & - & & - & 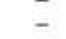 \\
\hline 705.70 & 44.3 & 77.6 & 0.1 & - & 9.3 & - & 2.0 & 1.6 & & 5.9 & & & & 3.4 & - & - & & - & - & - & - & - & - & & - & - \\
\hline 727.00 & 38.9 & 58.4 & - & - & 0.9 & - & 20.0 & 6.2 & & 1.3 & & & & 6.7 & - & 0.5 & & - & 4.9 & - & - & - & - & & 1.1 & - \\
\hline 744.80 & 44.7 & 73.1 & - & - & 1.0 & - & 13.1 & 2.9 & & - & & & & 6.1 & - & - & & 0.4 & - & 2.3 & - & 0.1 & - & & 0.9 & P \\
\hline 771.20 & 38.7 & 41.8 & - & - & 2.5 & - & 12.7 & 0.7 & & - & & & & 30.7 & - & - & & 9.6 & 1.3 & - & 0.7 & - & - & & - & P \\
\hline 830.30 & 15.5 & 95.1 & - & - & 1.5 & - & 2.2 & - & & 0.9 & & & & 0.4 & - & - & & - & - & - & - & - & - & & - & - \\
\hline \multicolumn{27}{|c|}{$2-20 \mu \mathrm{m}$ Fraction } \\
\hline 0.80 & 86.9 & & & & 22.3 & - & 5.7 & 20.4 & _. & 11.9 & 1.6 & - & _- & 6.0 & _- & _- & _ & - & - & 0.9 & _- & & 31.1 & - & _ & _- \\
\hline 2.70 & 92.2 & & & & 27.9 & - & 13.5 & 20.3 & - & 12.4 & 4.4 & - & - & -0 & - & 1.4 & - & - & - & - & - & & 20.0 & - & - & - \\
\hline 155.30 & 96.3 & & & & 13.3 & - & 4.6 & 7.0 & - & - & 4.4 & - & - & 8.1 & - & 2.8 & 15.3 & - & - & - & - & & 44.5 & - & - & - \\
\hline 268.30 & 95.2 & & & & 10.3 & - & 17.8 & 17.4 & - & - & - & - & - & - & - & - & - & - & - & - & - & & 54.5 & - & - & - \\
\hline 391.80 & 95.2 & & & & 13.1 & - & 31.9 & 20.8 & 5.1 & 13.5 & - & - & - & - & - & - & - & - & - & - & - & & 15.7 & - & - & - \\
\hline 448.60 & 95.8 & & & & 16.0 & - & 28.0 & 18.2 & - & 14.1 & - & - & - & - & - & - & - & - & 9.5 & - & - & & 14.1 & - & - & - \\
\hline 469.30 & 32.9 & & & & 13.4 & - & 14.8 & 9.8 & - & 5.2 & - & - & - & - & - & 42.0 & - & - & - & - & - & & 14.8 & - & - & - \\
\hline 486.50 & 32.4 & & & & 9.3 & - & 7.6 & 5.7 & - & 2.3 & - & - & - & - & - & 64.9 & - & - & - & - & - & & 10.2 & - & - & - \\
\hline 495.90 & -19.8 & & & & 14.7 & 80.0 & - & - & - & - & - & - & - & - & 5.4 & - & - & - & - & - & - & & - & - & - & - \\
\hline 515.40 & 66.1 & & & & 30.5 & - & 14.4 & 6.3 & - & 21.6 & 1.6 & - & 12.0 & 2.5 & - & - & - & - & - & - & - & & 11.0 & - & - & - \\
\hline 524.70 & 57.6 & & & & 30.3 & - & 15.7 & 2.3 & - & 23.1 & 1.2 & - & 18.1 & 1.4 & - & - & - & - & - & - & - & & 8.0 & $=$ & - & - \\
\hline 533.60 & 40.1 & & & & 40.2 & 5.3 & 13.7 & 6.3 & - & 18.7 & 1.0 & - & - & 0.9 & - & - & - & - & - & 0.1 & - & & 13.8 & - & - & - \\
\hline 553.30 & 49.6 & & & & 41.2 & - & 23.1 & 7.7 & - & 16.5 & 1.9 & - & - & - & - & - & - & - & - & 0.8 & - & & 0.8 & 7.9 & - & - \\
\hline 562.90 & 49.9 & & & & 33.6 & - & 24.6 & 7.1 & - & 16.5 & 1.2 & - & - & 2.1 & - & 0.5 & - & - & - & 0.2 & 0.2 & & 14.1 & - & - & - \\
\hline 571.80 & 50.0 & & & & 32.4 & - & 19.6 & 6.6 & 1.0 & 17.0 & - & - & 3.3 & 2.1 & - & - & - & - & - & 0.3 & - & & 17.6 & - & - & - \\
\hline 573.60 & 45.8 & & & & 38.0 & - & 9.2 & 6.0 & - & 17.7 & 0.9 & - & - & 4.0 & - & - & - & - & - & 0.8 & - & & 23.3 & - & - & - \\
\hline 583.10 & 38.7 & & & & 73.0 & - & - & - & - & 3.6 & - & - & - & - & - & 23.4 & - & - & - & - & - & & - & - & - & - \\
\hline 584.40 & 43.6 & & & & 46.8 & - & 12.9 & 8.4 & - & 11.3 & - & - & - & 0.6 & - & 19.9 & - & - & - & - & - & & - & - & - & - \\
\hline 587.40 & 44.6 & & & & 57.6 & - & 12.8 & 8.3 & - & 17.8 & 1.5 & - & - & 1.3 & - & 0.7 & - & - & - & - & - & & - & - & - & - \\
\hline 609.80 & 45.4 & & & & 43.8 & - & 14.9 & 7.2 & - & 13.6 & 1.0 & - & - & 2.4 & - & 13.3 & - & - & - & - & - & & 4.0 & - & - & - \\
\hline 610.50 & 39.6 & & & & 1.7 & - & 2.6 & 1.2 & - & 3.6 & - & - & - & 81.0 & - & 6.2 & - & - & - & - & - & & - & - & 3.7 & P \\
\hline 60 & 43.7 & & & & 58.1 & - & 15.0 & 5.9 & 0.9 & 11.4 & - & - & - & - & - & - & - & - & 5.2 & - & - & & 3.5 & - & - & - \\
\hline 630.60 & 42.2 & & & & 27.0 & - & 7.6 & 3.3 & - & 8.8 & - & - & - & 44.2 & - & 8.2 & - & - & - & - & - & & 0.9 & - & - & P \\
\hline 638.40 & 35.6 & & & & 39.8 & - & 16.0 & 6.2 & 0.5 & 8.1 & - & - & - & 1.1 & - & 21.0 & - & - & 6.1 & - & - & & 1.4 & - & - & TR \\
\hline 641.80 & 34.7 & & & & 3.3 & - & 44.3 & 6.4 & - & 5.9 & - & - & 1.0 & 4.2 & - & 11.6 & - & - & 17.3 & - & - & & - & - & 6.0 & - \\
\hline
\end{tabular}




\begin{tabular}{|c|c|c|c|c|c|c|c|c|c|c|c|c|c|c|c|c|c|c|c|c|c|c|c|}
\hline 642.10 & 38.2 & 3.1 & - & 43.1 & 4.4 & - & 5.6 & - & 4.9 & - & 7.0 & - & 13.2 & - & - & 16.7 & - & - & & - & - & 1.9 & P \\
\hline 667.10 & 46.1 & 13.7 & - & 15.6 & 12.1 & - & 8.3 & - & - & - & 25.8 & - & 17.7 & - & - & 5.6 & - & - & & - & - & 1.4 & P \\
\hline 668.10 & 45.6 & 20.2 & - & 32.4 & 4.6 & 1.0 & 12.9 & - & - & - & 16.8 & - & 2.8 & - & - & 8.8 & - & - & & - & - & 0.5 & $\mathrm{TR}$ \\
\hline 686.70 & 38.4 & 45.7 & - & 27.2 & 6.8 & - & 14.8 & - & - & - & - & - & - & - & - & 4.6 & - & - & & - & - & 0.9 & - \\
\hline 704.50 & 33.0 & 7.3 & - & 68.8 & 3.2 & - & 7.2 & - & - & - & 2.4 & - & 1.4 & - & - & 6.8 & - & - & & - & - & 3.0 & - \\
\hline 727.00 & 28.9 & 5.1 & - & 57.0 & 7.6 & - & 4.5 & - & - & - & 1.6 & - & 3.5 & - & - & 13.9 & - & - & & 1.4 & - & 5.3 & - \\
\hline 744.80 & 37.7 & 5.2 & - & 61.9 & 6.6 & - & 3.0 & 1.0 & - & - & - & - & - & - & 0.5 & - & 7.5 & - & & - & - & 14.4 & - \\
\hline 771.20 & 22.9 & 7.9 & - & 36.9 & 3.6 & - & 4.9 & - & - & - & 38.0 & - & - & - & 7.8 & 0.8 & - & - & & - & - & - & P \\
\hline 830.30 & 17.3 & 26.4 & - & 58.0 & 5.4 & - & 8.5 & - & - & - & - & - & 1.8 & - & - & - & - & - & & - & - & - & - \\
\hline \multicolumn{24}{|c|}{$<2 \mu \mathrm{m}$ Fraction } \\
\hline 0.80 & 88.7 & 15.3 & - & 8.3 & 11.6 & 8.7 & 7.5 & - & - & - & 43.3 & - & - & - & - & - & - & - & 0.3 & 5.1 & & - & - \\
\hline 2.70 & 79.5 & 9.8 & - & 5.4 & 6.5 & 2.8 & 8.3 & 2.5 & - & - & 63.2 & - & - & - & - & - & - & - & 0.2 & 1.6 & & - & - \\
\hline 155.30 & 92.9 & 8.1 & - & 10.7 & 13.2 & - & 3.7 & 4.9 & - & - & 43.6 & - & - & - & - & - & - & 0.6 & 0.5 & 14.6 & & - & - \\
\hline 268.30 & 91.4 & 10.9 & - & 7.8 & 5.1 & - & - & 9.0 & - & - & 58.0 & - & - & - & - & - & - & - & - & 9.4 & & - & - \\
\hline 391.80 & 81.6 & 5.9 & - & 7.3 & 7.8 & - & 8.1 & 2.1 & - & - & 51.8 & - & 1.1 & 11.5 & - & - & - & - & 0.3 & 4.2 & & - & - \\
\hline 448.60 & 79.1 & 5.2 & - & 9.2 & 6.0 & - & 10.8 & 1.7 & - & - & 60.6 & - & 0.9 & - & - & 1.8 & - & - & - & 3.8 & & - & - \\
\hline 469.30 & 75.6 & 13.0 & - & 10.6 & 6.9 & - & 5.6 & - & - & - & 51.5 & - & 2.9 & - & - & 4.6 & 0.8 & - & 0.2 & 3.8 & & - & - \\
\hline 486.50 & 35.3 & 1.8 & 60.7 & 1.1 & 0.8 & - & 6.5 & - & - & - & 25.5 & 1.9 & 1.0 & - & - & - & - & - & 0.1 & 0.8 & & - & - \\
\hline 495.90 & -11.4 & 1.5 & 82.2 & - & - & - & 0.4 & - & - & 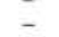 & - & 15.8 & 1.0 & $\overline{-}$ & 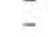 & 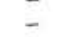 & 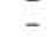 & 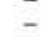 & - & - & & 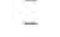 & - \\
\hline 515.40 & 76.1 & 11.0 & - & 2.8 & - & - & 14.6 & 3.4 & - & 21.3 & 43.3 & 15.0 & 1.2 & $\overline{-}$ & 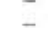 & $\overline{-}$ & $\overline{-}$ & $0 . \overline{6}$ & 1.8 & 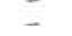 & & - & - \\
\hline 524.70 & $\begin{array}{l}70.1 \\
61.5\end{array}$ & $\begin{array}{r}1.0 \\
6.6\end{array}$ & 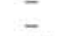 & 2.8 & $=$ & $\overline{-}$ & $\begin{array}{l}1.6 \\
22.4\end{array}$ & 3.4 & $=$ & 25.3 & $\begin{array}{l}45.3 \\
45.3\end{array}$ & $=$ & 1.2 & - & $\overline{-}$ & $=$ & 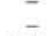 & ${ }^{0.6}$ & 0.4 & $=$ & & $\overline{-}$ & $\overline{-}$ \\
\hline 533.60 & 71.8 & 17.0 & 4.6 & 5.2 & 3.1 & - & 36.5 & 1.8 & - & - & 26.9 & - & 0.6 & - & - & 0.5 & 0.9 & 0.3 & 0.2 & 2.5 & & - & - \\
\hline 553.30 & 77.5 & 18.3 & - & 10.9 & 4.0 & - & 23.8 & 1.8 & - & - & 36.8 & - & - & - & - & 3.0 & 0.8 & 0.3 & 0.3 & - & & - & - \\
\hline 562.90 & 74.1 & 10.7 & - & 1.0 & 2.3 & - & 20.7 & 1.4 & - & - & 49.3 & - & - & 9.4 & - & 1.1 & 0.9 & 0.3 & 0.3 & 2.8 & & - & - \\
\hline 571.80 & 73.4 & 12.8 & - & 4.4 & 3.8 & - & 15.0 & 1.9 & - & 10.2 & 39.0 & - & 0.6 & 6.6 & - & - & - & 0.4 & - & 5.2 & & - & - \\
\hline 573.60 & 73.8 & 32.1 & - & 1.9 & 1.2 & - & 17.0 & 2.2 & - & - & 39.0 & - & - & - & - & - & 1.8 & 0.4 & 0.2 & 4.1 & & - & - \\
\hline 583.10 & 57.2 & 88.1 & - & - & - & - & 5.5 & - & - & - & - & - & 6.4 & - & - & - & - & - & - & - & & - & - \\
\hline 584.40 & 74.8 & 37.2 & - & 6.3 & 4.3 & - & 12.8 & - & - & - & 38.4 & - & 1.0 & - & - & - & - & - & - & - & & - & - \\
\hline 587.40 & 59.8 & 59.0 & - & 1.3 & - & - & 10.8 & - & - & - & 28.9 & - & - & - & - & - & - & - & - & - & & - & - \\
\hline 609.80 & 52.6 & 24.6 & - & 1.8 & 1.0 & - & 6.2 & - & - & - & 65.7 & - & 0.5 & - & - & - & - & - & 0.3 & - & & - & - \\
\hline 610.50 & 49.8 & 0.8 & - & - & - & - & - & 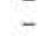 & 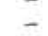 & 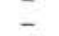 & 99.2 & - & 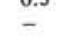 & - & - & 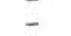 & 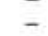 & - & - & 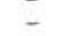 & & - & $P$ \\
\hline 613.60 & 56.4 & 51.3 & - & - & - & - & 8.4 & - & - & - & 40.1 & - & - & - & - & 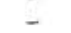 & - & - & 0.2 & - & & - & - \\
\hline 630.60 & 59.2 & 26.0 & - & 2.0 & 0.9 & - & 3.8 & - & - & - & 66.5 & - & 0.5 & - & - & - & - & - & 0.2 & - & & - & $P$ \\
\hline 638.40 & 55.6 & 61.3 & - & 2.3 & 1.5 & - & 3.8 & - & - & - & 29.6 & - & 1.2 & - & - & - & - & - & 0.2 & - & & - & P \\
\hline 641.80 & 31.6 & 2.7 & - & 5.2 & 4.4 & - & 1.8 & - & - & - & 80.7 & - & 1.4 & - & - & 2.9 & - & - & 0.2 & - & & 0.7 & $\mathrm{P}$ \\
\hline 642.10 & 34.8 & 2.1 & - & 18.8 & 4.9 & - & 1.3 & - & - & - & 59.9 & - & 5.4 & - & - & 7.0 & - & - & 0.2 & - & & 0.8 & $\mathbf{P}$ \\
\hline 667.10 & 47.3 & 13.5 & - & 1.7 & 0.8 & - & 3.9 & - & - & - & 79.5 & - & - & - & - & - & - & - & 0.5 & - & & - & $\mathbf{P}$ \\
\hline 668.10 & 43.1 & 10.1 & - & 3.5 & 2.3 & - & 1.6 & - & - & - & 78.5 & - & 0.4 & - & - & 2.6 & - & - & 0.8 & - & & 0.7 & P \\
\hline 686.70 & 55.3 & 33.0 & - & 3.7 & 1.0 & - & 4.4 & - & - & - & 57.5 & - & - & - & - & - & - & - & 0.4 & - & & - & - \\
\hline 704.50 & 28.2 & 1.8 & - & 1.2 & 0.8 & - & - & - & - & - & 96.2 & - & - & - & - & - & - & - & 0.1 & - & & - & P \\
\hline 727.00 & 35.4 & 0.9 & - & 6.5 & 2.5 & - & - & - & 8.5 & - & 77.6 & - & 0.5 & - & - & 3.3 & - & - & 0.1 & - & & - & $\mathrm{P}$ \\
\hline 744.80 & 54.2 & - & - & 8.1 & 2.6 & - & - & - & - & - & 85.6 & - & - & - & 3.4 & - & - & - & 0.3 & - & & - & P \\
\hline 771.20 & 38.8 & 0.4 & - & - & - & - & - & - & - & - & 96.9 & - & - & - & 0.5 & 2.3 & - & - & - & - & & - & $P$ \\
\hline 830.30 & 65.7 & 22.7 & - & 13.8 & 5.1 & - & 58.2 & - & - & - & - & - & - & - & $\div$ & - & - & - & 0.2 & - & & - & - \\
\hline
\end{tabular}

${ }^{a}$ Undifferentiated feldspar.

${ }^{b} \mathrm{U}-2=$ Major peak located at $3.50 \AA$. This peak may be due to the presence of anatase. However, it may also be associated with the montmorillonite. 
TABLE 12

Results of X-Ray Diffraction Analysis, Hole 317A

\begin{tabular}{|c|c|c|c|c|c|c|c|c|c|c|c|c|c|c|c|c|c|c|c|c|c|c|c|c|c|c|}
\hline $\begin{array}{l}\text { Sample } \\
\text { Depth Belo } \\
\text { Sea Floor } \\
\text { (m) }\end{array}$ & Amor. & Feld. $^{a}$ & Calc. & Dolo. & Quar. & Cris. & $\mathrm{K}-\mathrm{Fe}$. & Plag. & Kaol. & Mica & Chlo. & Paly. & Mont. & Trid. & Clin. & Phil. & Anal. & Hema. & Pyri. & Gibb. & Gyps. & Apat. & Bari. & Amph. & Magn. & Sani. $^{b}$ \\
\hline \multicolumn{27}{|l|}{ Bulk Sample } \\
\hline 554.20 & 41.2 & & 82.9 & - & 2.5 & - & 1.5 & 0.8 & - & 3.0 & - & - & - & - & 7.8 & & - & - & - & & - & - & 1.5 & - & & - \\
\hline 564.50 & 7.4 & & 97.8 & - & 2.2 & - & - & - & - & - & - & - & - & - & - & & - & - & - & & - & - & - & - & & - \\
\hline 576.90 & 52.5 & & 78.2 & - & 8.3 & - & 7.3 & - & - & 5.9 & - & - & - & - & - & & - & - & - & & 0.4 & - & - & - & & - \\
\hline 585.00 & 56.9 & & - & - & 9.7 & - & 10.8 & - & - & - & 0.9 & 63.3 & 7.8 & - & 1.4 & & - & - & - & & - & 6.0 & - & - & & - \\
\hline 602.40 & 7.1 & & 43.7 & 0.3 & 5.9 & 42.2 & - & - & - & - & - & - & - & 5.5 & - & & - & - & 0.1 & & 0.2 & - & 2.0 & - & & - \\
\hline 623.30 & -6.2 & & 34.3 & - & 0.6 & 55.5 & 0.9 & 0.4 & - & - & - & - & 1.5 & 4.3 & 1.2 & & - & - & - & & - & - & 0.5 & 0.7 & & - \\
\hline 644.10 & 28.7 & & 78.7 & - & 0.6 & - & 4.8 & 3.3 & - & - & - & - & 6.0 & - & 3.2 & & - & - & - & & - & 0.3 & 1.5 & 1.5 & & - \\
\hline 651.00 & 44.2 & & - & - & 2.1 & - & - & 16.0 & 0.6 & - & - & - & 71.8 & - & 9.5 & & - & - & - & & - & - & - & - & & - \\
\hline 670.00 & 13.2 & & - & - & 0.2 & - & - & - & - & - & - & - & 76.3 & - & - & & 23.4 & - & - & & - & - & - & - & & - \\
\hline 698.60 & 52.5 & & - & - & - & - & - & - & - & - & - & - & 58.6 & - & - & & 12.4 & - & - & & - & - & - & - & & 29.1 \\
\hline 727.70 & 48.3 & & - & - & 2.3 & - & 17.1 & 11.1 & - & - & - & - & 69.5 & - & - & & - & - & - & & - & - & - & - & & - \\
\hline 755.90 & 52.7 & & - & - & 2.8 & - & 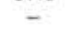 & - & - & - & 2.3 & - & 90.4 & - & - & & - & 4.6 & - & & - & - & - & - & & - \\
\hline 756.70 & 58.2 & & - & - & - & - & - & - & - & - & - & - & 91.7 & - & - & & 2.4 & 0.6 & - & & - & - & - & - & & 5.2 \\
\hline 757.40 & 51.0 & & 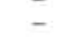 & - & 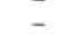 & - & 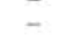 & - & 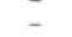 & 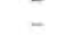 & $=$ & 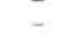 & 95.6 & 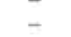 & 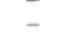 & & 0.5 & - & 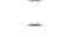 & & 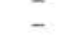 & 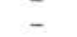 & - & 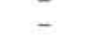 & & 3.8 \\
\hline 759.40 & 62.8 & & - & - & - & - & - & - & - & - & - & - & 80.1 & - & - & & 11.1 & - & - & & - & - & - & - & & 8.9 \\
\hline 777.70 & 43.0 & & - & - & - & - & $=$ & - & - & - & - & - & 92.8 & - & - & & 2.5 & - & - & & 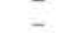 & - & - & - & & $\begin{array}{l}0.7 \\
4.7\end{array}$ \\
\hline 778.10 & 58.8 & & 4.5 & - & - & - & - & - & - & - & - & - & 84.5 & - & - & & 2.8 & 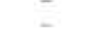 & 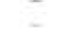 & & 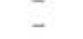 & 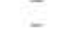 & - & 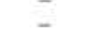 & & 8.3 \\
\hline 779.60 & 48.5 & & - & - & - & - & - & - & - & - & - & - & 88.3 & - & & & 4.1 & - & - & & - & - & - & - & & 7.6 \\
\hline 821.10 & 49.1 & & - & - & - & - & - & - & - & - & - & & 88.8 & & & & 6.4 & - & - & & - & - & - & - & & 4.8 \\
\hline 849.90 & 44.8 & & 1.4 & - & - & - & - & - & - & - & - & - & 68.7 & & & & 25.7 & - & - & & - & - & - & - & & 4.2 \\
\hline 887.40 & 25.1 & & 0.4 & - & - & - & - & - & - & - & - & - & 92.3 & .. & & & 5.7 & - & - & & - & - & - & - & & 1.5 \\
\hline 887.40 & 39.4 & &.- & - & - & - & - & - & - & 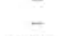 & - & & 94.8 & & - & & 3.8 & 1.4 & - & & - & - & - & - & & - \\
\hline 927.50 & 47.9 & & - & - & 8.1 & - & 8.7 & 36.1 & - & 10.5 & & & 13.4 & & 10.0 & & - & 13.2 & - & & - & - & - & - & & - \\
\hline \multicolumn{27}{|c|}{$2.20 \mu \mathrm{m}$ Fraction } \\
\hline 554.20 & 30.1 & - & & & 16.6 & - & 9. & 5.9 & - & 9.0 & - & - & 1.0 & - & 45.0 & 3.8 & 0.8 & & - & & _- & - & 8.3 & _- & - & - \\
\hline 564.50 & 24.0 & - & & & 43.0 & - & 47.8 & 4.0 & - & 5.2 & - & & $\cdots$ & -- & - & - & - & - & - & & - & - & - & - & - & - \\
\hline 576.90 & 31.4 & - & & & 34.6 & - & 42.6 & 1.1 & - & 20.0 & 0.8 & - & - & & - & - & - & - & - & & - & - & - & 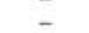 & 0.9 & - \\
\hline 585.00 & 49.7 & - & & & 20.7 & - & 23.7 & 2.8 & - & 21.0 & 1.7 & 4.1 & 10.5 & - & 6.9 & & - & - & - & & 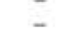 & 8.6 & - & $\overline{-}$ & 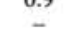 & - \\
\hline 602.40 & -12.9 & - & & & 25.1 & 56.6 & 1.1 & 0.7 & - & - & - & & & 5.4 & - & - & - & - & - & & - & - & 11.1 & - & - & - \\
\hline 623.30 & -33.4 & - & & & 2.3 & 75.8 & 6.1 & 2.1 & - & - & - & & 0.9 & 8.6 & 0.7 & & - & - & & & - & 0.7 & 1.5 & 1.3 & - & - \\
\hline 644.10 & 21.2 & - & & & 3.1 & - & 23.3 & 15.2 & - & - & - & - & 3.3 & -0 & 33.8 & & _- & - & - & & - & 1.6 & 9.5 & 10.2 & - & - \\
\hline 651.00 & 49.1 & - & & & 5.7 & - & - & 54.8 & 0.5 & - & - & - & 37.0 & - & 2.0 & - & & - & - & & - & $=$ & - & 10. & - & - \\
\hline 670.00 & 3.0 & - & & & 0.3 & - & 1.7 & - & 0. & 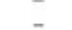 & .. & - & 52.1 & 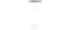 & 2.0 & & 45.9 & 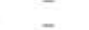 & - & & 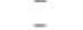 & $=$ & $=$ & 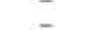 & - & 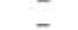 \\
\hline 698.60 & 45.6 & - & & & - & - & - & - & $=$ & - & - & - & 56.1 & & - & & 22.9 & 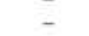 & - & & - & - & 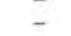 & - & - & 20.9 \\
\hline 727.00 & 63.7 & - & & & 3.7 & - & 46.2 & 20.9 & - & - & - & - & 29.1 & - & - & - & - & - & - & & - & - & - & - & - & - \\
\hline 755.90 & 59.1 & 6.5 & & & 4.4 & - & - & - & - & - & - & - & $\begin{array}{l}29.1 \\
83.6\end{array}$ & 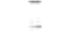 & 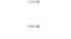 & 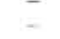 & $\overline{-}$ & 5.5 & $=$ & & 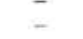 & $\overline{-}$ & 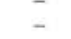 & $\overline{-}$ & $\overline{-}$ & $\overline{-}$ \\
\hline 756.70 & 64.6 & - & & & - & - & - & - & - & - & - & & 87.3 & - & $=$ & - & & 5.5 & - & & - & - & - & $=$ & - & 7.1 \\
\hline 757.40 & 66.6 & - & & & - & - & - & - & - & - & -- & - & 76.5 & - & - & - & 3.7 & - & - & & - & - & - & - & - & 19.3 \\
\hline 759.40 & 62.0 & - & & & - & - & - & - & - & - & - & - & 91.5 & - & - & - & - & - & - & & - & - & - & - & - & 8.5 \\
\hline 777.70 & 64.8 & - & & & - & - & - & - & - & - & - & - & 66.6 & - & - & - & 7.6 & - & - & & - & - & - & - & - & 25.8 \\
\hline $778.10^{\mathrm{c}}$ & 58.0 & - & & & - & - & 8.1 & - & - & - & - & - & 81.6 & - & 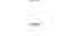 & - & 3.0 & - & - & & - & - & - & - & - & 7.3 \\
\hline 779.60 & 62.4 & - & & & - & - & - & 0.7 & - & - & - & - & 64.1 & 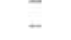 & 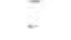 & 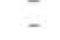 & 4.8 & 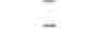 & - & & - & $\overline{-}$ & - & 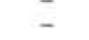 & 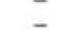 & 30.3 \\
\hline 821.10 & 58.5 & - & & & - & - & - & - & - & 1.8 & 1.0 & - & 73.8 & - & - & - & 6.9 & - & - & & - & - & - & - & - & 16.5 \\
\hline 849.90 & 56.6 & - & & & - & - & - & 2.9 & - & - & - & - & 40.2 & 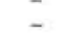 & 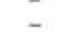 & - & 17.4 & - & 0.7 & & 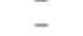 & - & 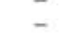 & - & 0.9 & 37.9 \\
\hline 887.40 & 35.2 & - & & & - & - & - & 1.2 & - & - & - & - & 30.1 & - & - & - & 54.3 & - & - & & - & - & - & - & - & 14.5 \\
\hline 887.40 & 60.9 & - & & & - & - & - & - & - & - & 1.1 & - & 76.6 & - & - & - & 8.7 & 0.6 & - & & - & - & - & - & - & 13.0 \\
\hline 927.50 & 39.1 & - & & & 13.4 & - & 10.0 & 45.3 & - & 5.6 & - & - & 4.3 & - & 10.3 & - & - & 10.6 & 0.4 & & - & - & - & - & - & - \\
\hline
\end{tabular}




\begin{tabular}{|c|c|c|c|c|c|c|c|c|c|c|c|c|c|c|c|c|c|c|c|c|c|}
\hline \multicolumn{22}{|c|}{$<2 \mu \mathrm{m}$ Fraction } \\
\hline 554.20 & 72.2 & - & 8.7 & - & 11.4 & 7.4 & 31.7 & & & 39.1 & - & 0.6 & - & - & - & 0.3 . & - & 0.8 & - & - & - \\
\hline 564.50 & 48.2 & - & 36.3 & - & 35.6 & 2.3 & 24.1 & 1.8 & & & - & - & - & - & - & - & - & - & - & - & - \\
\hline 576.90 & 78.6 & - & 25.6 & - & 23.9 & 1.6 & 27.6 & 2.0 & & 18.3 & - & - & - & - & - & - & - & - & - & 1.0 & - \\
\hline 585.00 & 48.7 & - & 3.6 & - & 2.2 & & 7.7 & 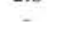 & 66.7 & 17.3 & - & 0.2 & - & - & 0.1 & - & 1.9 & 0.3 & - & - & - \\
\hline 602.40 & -19.6 & - & 2.4 & 83.4 & 0.4 & 0.2 & $\cdots$ & - & - & 1.8 & 10.9 & - & - & - & - & - & - & 0.8 & - & - & - \\
\hline 623.30 & -23.9 & - & 0.2 & 83.0 & 1.0 & 0.6 & & - & & 2.9 & 10.2 & 0.2 & - & - & - & - & 0.5 & 1.0 & 0.4 & - & - \\
\hline 644.10 & 33.9 & - & 2.3 & - & 9.1 & 6.1 & - & 0.2 & - & 78.3 & 10.2 & 2.0 & 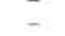 & - & $=$ & - & 0.3 & -0 & 1.6 & - & $\overline{-}$ \\
\hline 651.00 & 44.4 & - & - & - & - & 6.5 & - & - & - & 93.3 & - & 2.0 & z & 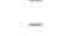 & $=$ & 0.2 & - & $=$ & 1.0 & $=$ & 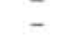 \\
\hline 670.00 & 22.8 & - & - & - & - & - & - & - & - & 86.4 & - & - & 13.6 & - & - & - & - & - & - & - & - \\
\hline 698.60 & 49.2 & - & - & - & - & - & 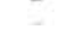 & - & - & $\begin{array}{l}80.4 \\
88.9\end{array}$ & 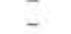 & 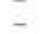 & 3.4 & $\overline{-}$ & $z$ & 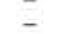 & $z$ & - & 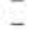 & - & 7.7 \\
\hline 727.00 & 48.4 & 5.1 & 0.4 & - & - & 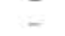 & - & 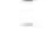 & - & $\begin{array}{l}90.7 \\
94.5\end{array}$ & 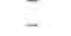 & 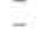 & 3.4 & - & 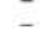 & 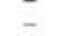 & 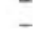 & 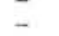 & 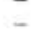 & $=$ & - \\
\hline 755.90 & 45.1 & - & - & - & - & - & - & & - & 96.7 & - & & - & 3.1 & - & 0.2 & - & - & - & - & - \\
\hline 756.70 & 41.6 & - & - & - & - & - & - & - & - & 97.1 & - & - & - & 2.9 & - & - & - & - & - & - & - \\
\hline 757.40 & 34.0 & - & - & - & - & - & - & - & - & 97.7 & - & - & - & - & - & - & - & - & - & - & 2.3 \\
\hline 759.40 & 90.6 & - & - & - & - & - & - & - & - & 96.6 & - & - & - & - & - & - & - & - & - & - & 3.4 \\
\hline 777.70 & 40.9 & 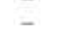 & - & - & - & - & - & - & 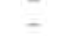 & 96.2 & - & 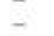 & 0.6 & 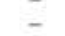 & 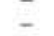 & - & $\overline{-}$ & 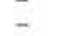 & 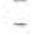 & 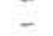 & 3.2 \\
\hline 778.10 & 32.6 & - & - & - & - & - & - & - & & 100.0 & - & - & - & - & - & - & - & - & - & - & - \\
\hline 779.60 & 41.5 & - & - & - & - & - & - & - & - & 95.2 & - & - & - & - & - & - & - & - & - & - & 4.8 \\
\hline 821.10 & 86.3 & - & - & - & - & - & - & - & - & 94.2 & - & - & - & - & - & - & - & $=$ & - & - & 5.8 \\
\hline 849.90 & 81.7 & - & - & - & - & 1.2 & - & - & - & 82.4 & - & - & 3.0 & - & - & - & - & - & - & - & 13.3 \\
\hline 887.40 & 84.0 & - & - & - & - & - & - & - & - & 95.6 & - & - & 3.1 & - & - & - & - & - & - & - & 1.2 \\
\hline 887.40 & 42.0 & - & - & - & - & - & - & - & - & 92.7 & - & - & - & 7.3 & - & - & - & - & - & - & - \\
\hline 927.50 & 48.9 & - & 3.4 & - & 5.3 & 18.2 & 18.0 & - & - & 39.2 & - & 0.8 & - & 15.1 & - & - & - & - & - & - & - \\
\hline
\end{tabular}

${ }^{\mathrm{a}}$ Undifferentiated feldspar.

The abbreviation sani, is for sanidine.

'Sample contains two types of $\mathrm{K}$-feldspar. They have been reported separately as K-spar and sanidine. 
TABLE 13

Results of X-Ray Diffraction Analysis, Hole 317B

\begin{tabular}{|c|c|c|c|c|c|c|c|c|c|c|c|c|c|c|c|c|c|c|c|c|c|}
\hline $\begin{array}{l}\text { Sample } \\
\text { Depth Below } \\
\text { Sea Floor } \\
\text { (m) }\end{array}$ & Amor. & Calc. & Dolo. & Arag. & Feld. & Cris. & $\mathrm{K}$-Fe. & Plag. & Kaol. & Mica & Chlo. & Mont. & Trid. & Clin. & Gibb. & Quar. & Gyps. & Apat. & Bari. & Psil. $^{\mathrm{a}}$ & Magn. \\
\hline \multicolumn{22}{|l|}{ Bulk Sample } \\
\hline $\begin{array}{r}0.30 \\
27.40 \\
65.70 \\
102.20 \\
141.20 \\
179.50 \\
220.70 \\
227.10 \\
266.80 \\
312.40 \\
352.30 \\
389.40\end{array}$ & $\begin{array}{l}23.4 \\
21.1 \\
26.5 \\
20.3 \\
17.3 \\
23.7 \\
35.7 \\
21.8 \\
29.5 \\
29.5 \\
17.4 \\
28.9\end{array}$ & $\begin{array}{r}100.0 \\
100.0 \\
100.0 \\
100.0 \\
100.0 \\
98.1 \\
99.3 \\
100.0 \\
100.0 \\
100.0 \\
100.0 \\
99.8\end{array}$ & $\begin{array}{l}- \\
- \\
- \\
- \\
- \\
\overline{0} \\
- \\
- \\
- \\
- \\
-\end{array}$ & $\begin{array}{l}- \\
- \\
- \\
\overline{-} \\
1.2 \\
- \\
- \\
- \\
- \\
-\end{array}$ & $\begin{array}{l}- \\
- \\
- \\
- \\
\overline{0} \\
- \\
- \\
- \\
- \\
- \\
-\end{array}$ & & & & & & & & & & & $\begin{array}{l}- \\
- \\
- \\
- \\
\overline{0} \\
0.1 \\
- \\
- \\
- \\
\overline{0} \\
0.2\end{array}$ & $\begin{array}{l}- \\
- \\
- \\
- \\
\overline{-} \\
0.2 \\
- \\
\overline{-} \\
- \\
-\end{array}$ & & & & \\
\hline \multicolumn{22}{|c|}{ 2-20 $\mu$ m Fraction } \\
\hline $\begin{array}{r}0.30 \\
27.40 \\
65.70 \\
102.20 \\
141.20 \\
179.50 \\
220.70 \\
227.10 \\
266.80 \\
352.30 \\
389.40\end{array}$ & $\begin{array}{c}31.0 \\
10.5 \\
32.5 \\
80.8 \\
89.4 \\
91.9 \\
- \\
94.8 \\
2.6 \\
32.3 \\
41.8\end{array}$ & & & & & $\begin{array}{l}- \\
- \\
- \\
- \\
\overline{-} \\
- \\
- \\
\bar{y} \\
\overline{3} .3\end{array}$ & $\begin{array}{r}23.5 \\
39.4 \\
27.8 \\
8.4 \\
34.9 \\
29.9 \\
\overline{7.0} \\
21.6 \\
19.4 \\
21.3\end{array}$ & $\begin{array}{r}29.3 \\
15.4 \\
10.4 \\
26.9 \\
8.1 \\
11.3 \\
-\bar{y} \\
28.1 \\
12.3 \\
11.2 \\
7.8\end{array}$ & & $\begin{array}{c}- \\
- \\
- \\
\overline{-} \\
10.4 \\
- \\
\overline{-} \\
22.7 \\
18.4 \\
6.9 \\
1.7\end{array}$ & $\begin{array}{l}- \\
- \\
- \\
- \\
- \\
- \\
\overline{2.7} \\
- \\
- \\
-\end{array}$ & $\begin{array}{c}- \\
\overline{-} \\
\overline{-} \\
1 \overline{2.2} \\
\overline{-} \\
\overline{-} \\
\overline{3} \\
3.1 \\
10.6\end{array}$ & & $\begin{array}{l}- \\
- \\
\overline{2.1} \\
\overline{-} \\
\overline{-} \\
\overline{-} \\
32.4 \\
25.7 \\
39.9\end{array}$ & & $\begin{array}{r}7.8 \\
5.5 \\
6.2 \\
2.1 \\
9.2 \\
11.3 \\
- \\
22.7 \\
15.3 \\
9.1 \\
2.5\end{array}$ & & & $\begin{array}{c}39.5 \\
39.7 \\
55.5 \\
60.5 \\
25.2 \\
47.5 \\
\overline{1} \\
16.8 \\
\overline{-} \\
24.7 \\
13.0\end{array}$ & $\begin{array}{c}- \\
- \\
- \\
- \\
- \\
\overline{100.0} \\
- \\
- \\
- \\
-\end{array}$ & \\
\hline \multicolumn{22}{|c|}{$<2 \mu$ m Fraction } \\
\hline $\begin{array}{r}0.30 \\
27.40 \\
65.70 \\
102.20 \\
141.20 \\
179.50 \\
220.70 \\
227.10 \\
266.80 \\
312.40 \\
352.30 \\
389.40\end{array}$ & $\begin{array}{l}90.2 \\
74.9 \\
73.0 \\
84.5 \\
74.2 \\
66.5 \\
93.8 \\
84.9 \\
69.5 \\
70.5 \\
84.9 \\
45.2\end{array}$ & & & & & $\begin{array}{l}- \\
= \\
= \\
= \\
= \\
- \\
= \\
= \\
54.1\end{array}$ & $\begin{array}{r}14.7 \\
12.7 \\
6.0 \\
14.6 \\
7.6 \\
3.5 \\
- \\
- \\
4.6 \\
2.3 \\
8.1 \\
0.8\end{array}$ & $\begin{array}{r}22.7 \\
5.5 \\
2.7 \\
7.0 \\
3.7 \\
2.7 \\
- \\
- \\
3.0 \\
1.1 \\
5.3 \\
0.5\end{array}$ & $\begin{array}{l}4.4 \\
- \\
- \\
- \\
- \\
1.0 \\
- \\
- \\
1.1 \\
- \\
- \\
-\end{array}$ & $\begin{array}{c}- \\
\overline{-} \\
\overline{9} .7 \\
6.4 \\
4.4 \\
\overline{19.7} \\
15.3 \\
15.9 \\
9.8 \\
4.8\end{array}$ & $\begin{array}{l}- \\
- \\
\overline{5.1} \\
2.3 \\
- \\
- \\
\overline{-} \\
\overline{3.6} \\
\overline{-}\end{array}$ & $\begin{array}{c}29.2 \\
73.6 \\
73.0 \\
11.5 \\
72.0 \\
79.9 \\
- \\
64.1 \\
65.8 \\
71.0 \\
58.9 \\
31.4\end{array}$ & $\begin{array}{l}- \\
- \\
- \\
- \\
- \\
- \\
- \\
- \\
- \\
- \\
4.5\end{array}$ & $\begin{array}{l}- \\
- \\
\overline{1} \\
1.6 \\
- \\
- \\
- \\
\overline{-} \\
5.1 \\
3.4 \\
6.2 \\
0.9\end{array}$ & $\begin{array}{l}- \\
- \\
- \\
1.5 \\
- \\
- \\
- \\
- \\
- \\
- \\
- \\
-\end{array}$ & $\begin{array}{l}6.8 \\
1.0 \\
1.3 \\
4.4 \\
2.6 \\
1.8 \\
- \\
9.6 \\
5.1 \\
2.9 \\
5.9 \\
3.0\end{array}$ & $\begin{array}{l}- \\
0.4 \\
0.3 \\
-\overline{0} \\
0.4 \\
2.3 \\
- \\
- \\
- \\
- \\
-\end{array}$ & $\begin{array}{c}- \\
\overline{7.6} \\
- \\
- \\
- \\
- \\
- \\
- \\
- \\
- \\
-\end{array}$ & $\begin{array}{r}22.2 \\
6.9 \\
7.5 \\
44.4 \\
4.8 \\
6.3 \\
- \\
6.6 \\
- \\
- \\
5.9 \\
-\end{array}$ & & $\begin{array}{l}- \\
\overline{1} \\
\overline{-} \\
- \\
- \\
- \\
- \\
- \\
- \\
- \\
-\end{array}$ \\
\hline
\end{tabular}

${ }^{\mathrm{a}}$ The abbreviation Psil. is for psilomelene. 
TABLE 14

Results of X-Ray Diffraction Analysis, Site 318

Sample

Depth Below

Sea Floor

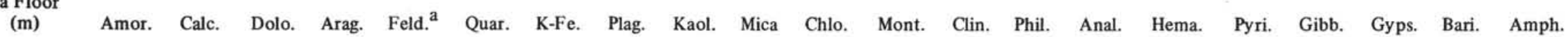

\begin{tabular}{|c|c|c|c|c|c|c|c|c|c|c|c|c|c|c|c|c|c|c|c|c|c|}
\hline \multicolumn{22}{|l|}{ Bulk Sample } \\
\hline 1.30 & 39.4 & 80.4 & 1.0 & 18.6 & - & - & - & - & & - & & - & - & & & - & - & & - & - & - \\
\hline 93.70 & 27.0 & 99.5 & 0.5 & - & - & - & - & - & & - & & - & - & & & - & - & & - & - & - \\
\hline 122.50 & 31.0 & 99.6 & 0.4 & - & - & - & - & - & & - & & - & - & & & - & - & & - & - & - \\
\hline 179.80 & 25.2 & 100.0 & - & - & - & - & - & - & & - & & - & - & & & - & - & & - & - & - \\
\hline 266.50 & 30.3 & 100.0 & - & - & - & - & - & - & & - & & - & - & & & - & - & & - & - & - \\
\hline 350.80 & 30.8 & 100.0 & - & - & - & - & - & - & & - & & - & - & & & - & - & & - & - & - \\
\hline 407.50 & 25.0 & 100.0 & - & - & - & - & - & - & & - & & - & - & & & - & - & & - & - & - \\
\hline 492.70 & 29.7 & 99.5 & 0.3 & - & - & 0.2 & - & - & & - & & - & - & & & - & - & & - & - & - \\
\hline 578.50 & 27.2 & 98.9 & - & - & 0.9 & 0.2 & - & - & & - & & - & - & & & - & - & & - & - & - \\
\hline 581.10 & 34.7 & 94.0 & - & - & 1.2 & 0.3 & - & - & & - & & - & 4.5 & & & - & - & & - & - & - \\
\hline 615.80 & 10.2 & 31.6 & - & - & - & 2.1 & 11.6 & 4.1 & & 1.3 & & 43.1 & 6.2 & & & - & - & & - & - & - \\
\hline 630.70 & 45.7 & 13.5 & - & - & - & 6.8 & 11.8 & 4.7 & & - & & 63.2 & - & & & - & - & & - & - & - \\
\hline 665.60 & 69.4 & 5.6 & - & - & - & 0.6 & 9.4 & 13.2 & & - & & 68.3 & 1.3 & & & 1.6 & - & & - & - & - \\
\hline 703.80 & 58.3 & 4.8 & - & - & - & 2.0 & 55.4 & 10.1 & & - & & 12.2 & 14.8 & & & - & - & & - & - & 0.7 \\
\hline 743.50 & 41.2 & 2.96 & - & - & - & 1.0 & 9.1 & 4.4 & & 1.1 & & 33.5 & 20.4 & & & - & 0.4 & & - & - & 0.6 \\
\hline \multicolumn{22}{|c|}{ 2-20 $\mu \mathrm{m}$ Fraction } \\
\hline 1.30 & 48.7 & & 59.6 & & & 1.5 & 5.9 & 11.0 & - & - & 1.6 & - & 0.7 & 11.1 & - & 4.5 & 0.8 & & & 3.2 & - \\
\hline 179.80 & 91.3 & & - & & & 2.3 & 6.5 & 9.3 & - & - & - & - & - & - & - & - & - & & & 81.9 & - \\
\hline 266.50 & 94.7 & & - & & & 10.0 & 26.3 & 9.8 & - & 21.0 & - & - & - & - & - & - & - & & & 33.0 & - \\
\hline 350.80 & 95.1 & & - & & & 25.8 & 7.8 & 24.8 & - & 24.2 & - & - & - & - & - & - & - & & & 17.4 & - \\
\hline 407.50 & 50.1 & & - & & & 16.4 & 17.4 & 8.8 & - & 11.3 & - & 5.7 & 33.7 & - & 0.8 & - & - & & & 5.9 & - \\
\hline 492.70 & 56.7 & & - & & & 2.2 & 14.8 & 9.6 & - & 2.2 & - & 8.3 & 40.1 & - & - & 12.6 & 5.5 & & & 4.7 & - \\
\hline 578.50 & 56.9 & & - & & & 4.4 & 22.8 & 14.8 & - & - & 0.8 & 16.5 & 31.0 & - & 0.7 & - & - & & & 8.9 & - \\
\hline 581.10 & 33.7 & & - & & & 3.9 & 17.1 & 9.8 & - & - & - & 5.8 & 58.7 & - & - & - & - & & & 4.8 & - \\
\hline 615.80 & 38.1 & & - & & & 5.0 & 19.7 & 15.3 & - & 0.6 & - & 26.8 & 31.6 & - & - & - & 1.0 & & & - & - \\
\hline 630.70 & 72.9 & & - & & & 10.6 & 22.1 & 17.8 & - & - & - & 49.4 & - & - & - & - & - & & & - & - \\
\hline 665.60 & 43.4 & & - & & & 1.3 & 48.7 & 4.6 & - & - & - & 38.4 & 5.7 & - & - & 0.9 & 0.4 & & & - & - \\
\hline 703.80 & 46.4 & & - & & & 3.0 & 21.3 & 13.1 & - & - & 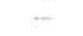 & 18.4 & 42.9 & - & - & 1.0 & - & & & - & 0.4 \\
\hline 743.50 & 37.1 & & - & & & 0.5 & 63.7 & 18.9 & - & 0.6 & - & 12.5 & 3.3 & - & - & - & - & & & - & 0.4 \\
\hline \multicolumn{22}{|c|}{$<2 \mu \mathrm{m}$ Fraction } \\
\hline 1.30 & 96.0 & & & & & 3.1 & 17.7 & 17.3 & & & & 52.4 & - & & & - & - & - & - & 9.6 & \\
\hline 93.70 & 87.8 & & & & & 5.3 & 11.9 & 12.9 & & & & 58.6 & - & & & - & - & - & - & 11.4 & \\
\hline $122.50^{\mathrm{b}}$ & - & & & & & - & - & - & & & & - & - & & & - & - & - & - & - & \\
\hline 179.80 & 90.1 & & & & & - & - & - & & & & 61.5 & - & & & - & - & - & - & 38.5 & \\
\hline 266.50 & 89.9 & & & & & 3.2 & 10.8 & 7.0 & & & & 68.9 & - & & & - & - & - & - & 10.1 & \\
\hline 350.80 & 89.5 & & & & & 6.6 & 7.4 & 5.8 & & & & 74.9 & - & & & - & - & - & - & 5.3 & \\
\hline 407.50 & 73.3 & & & & & 1.2 & 2.6 & 2.1 & & & & 92.1 & 2.0 & & & - & - & - & - & - & \\
\hline 492.70 & 78.4 & & & & & 2.3 & 6.1 & 4.5 & & & & 76.4 & 4.6 & & & - & 2.2 & 0.7 & 0.4 & 2.8 & \\
\hline 578.50 & 79.2 & & & & & 2.2 & 11.0 & 7.2 & & & & 67.3 & 6.0 & & & - & - & - & - & - & \\
\hline 581.10 & 64.0 & & & & & - & - & - & & & & 98.6 & 1.1 & & & - & - & - & 0.3 & - & \\
\hline 615.80 & 71.0 & & & & & 1.4 & 15.6 & 8.9 & & & & 65.4 & 5.7 & & & 3.0 & - & - & - & - & \\
\hline 630.70 & 64.3 & & & & & - & - & - & & & & 99.8 & - & & & - & - & - & 0.2 & - & \\
\hline 665.60 & 71.3 & & & & & 0.5 & 16.5 & 12.2 & & & & 69.3 & 0.7 & & & 0.1 & 0.7 & - & - & - & \\
\hline 703.80 & 68.1 & & & & & - & 7.4 & 4.8 & & & & 85.4 & 1.4 & & & - & 1.0 & - & - & - & \\
\hline 743.50 & 41.3 & & & & & - & 3.1 & 2.0 & & & & 94.2 & 0.6 & & & - & - & - & - & - & \\
\hline
\end{tabular}

bominant constituant was tungsten carbide contaminant. Sample also contained minor amounts of pyrite and goethite. 
TABLE 15

Samples Used in X-Ray Diffraction Analysis, Leg 33 (Composited samples are bracketed)

\begin{tabular}{cl}
\hline Sample & Depth Below \\
(Interval in cm) & Sea Floor (m)
\end{tabular}

Site 314

$3, \mathrm{CC}$

$\begin{array}{ll}3, \mathrm{CC} & 0-45 \\ \mathrm{X} & 0-45\end{array}$

\section{Hole 315}

$1-1,130-132$

$1-1,135-137$

$1-2,20-22$

$1-2,88-90$

$1-3,85-87$

4-1, 143-145

4-2, 121-123

4-2, 135-137

$4-3,56-58$

$4-3,85-87$

$4-4,87-89$

4-4, $116-118$

$4-5,7-9$

4-5, 130-132

$4-6,148-150$

$0-45$

\section{Hole 315A}

$1-1,108-110$

$1-5,90-92$

$1-5,95-97$

$1-6,136-138$

$1-6,142-144$

2-1, 140-142

2-1, 145-147

$3-1,40-42$

$3-1,68-70$

3-3, 70-72

3-3, 74-76

$3-5,70-72$

$3-5,138-140$

4-3, 103-105

4-3, 117-119

5-2, 59-61

5-2, 144-146

6-1, 87-89

6-1, 92-94

6-2, 128-130

6-2, 140-142

7-1, 94-96

7-1, 100-102

7-2, 69-71

7-2, 98-100

8-2, 97-99

8-2, 101-103

9-1, 108-110

9-1, 120-122

$10-1,52-54$

10-1, 70-72

10-1, 115-117

10-6, 100-102

$10-6,102-104$

$11-1,134-136$

$11-1,146-150$

$15-1,114-116$

$15-2,82-84$

16-1, 101-103

$17-1,7-9$

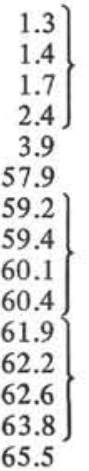

76.6

82.

83.0

84.9

124.9

125.0

142.6

142.9

145.9

146.0

148.9

149.6

260.0

260.2

372.1

373.0

465.9

465.9

467.8

467.9

513.5

513.5

514.7

515.0

591.0

$591.0\}$

703.6

703.7

731.3

731.4

732.0

739.3

739.3

741.4

$741.5\}$

779.2

779.6

788.5

797.1
TABLE 15 - Continued

\begin{tabular}{lc}
\hline $\begin{array}{c}\text { Sample } \\
\text { (Interval in cm) }\end{array}$ & $\begin{array}{c}\text { Depth Below } \\
\text { Sea Floor }(\mathrm{m})\end{array}$ \\
\hline $19-2,118-120$ & 818.7 \\
$19-3,52-53$ & 819.5 \\
$20-1,143-144$ & 826.9 \\
$20-2,96-97$ & 828.0 \\
$20-4,0-150$ & 830.0 \\
$21-2,32-34$ & 836.8 \\
$21-2,32-34$ & 836.8 \\
$21-2,46-47$ & 837.0 \\
$22-4,145-146$ & 850.5 \\
$23-2,49-51$ & 856.0 \\
$23-3,130-131$ & 858.3 \\
$24-2,136-137$ & 875.9 \\
$25-1,138-139$ & 893.4 \\
$25-2,72-74$ & 894.2 \\
$25-3,48-50$ & 895.5 \\
$26-1,26-28$ & 911.3 \\
$26-1,130-132$ & 912.3 \\
$27-2,119-120$ & 932.7 \\
$28-3,75-77$ & 952.8 \\
$28-3,85-87$ & 952.9 \\
$29-2,136-138$ & 970.9 \\
$30-2,148-150$ & 990.0 \\
\end{tabular}

Site 316

1-1, 83-85

$1-1,85-88$

1-2, 116-118

$1-2,118-120$

2-2, 80-82.5

2-2, 82.5-85

$3-1,126-128$

3-2, 72-74

4-1, 124-128

4-1, 128-131

5-1, 110-115

5-2, 101-104

7-2, 133-135

9-1, 100-102

10-1, 91-93

11-1, 135-138

12-1, 123-126

$12-2,15-17$

13-1, 56-59

$15-1,101-103$

$16-1,137-140$

$17-1,77-80$

17-2, 104-106

18-2, 109-111

$18-3,96-99$

18-5, 84-86

19-1, 83-85

19-1, 146-150

$19-4,10-14$

20-2, 112-114

21-1, $92-96$

21-3, 25-29

21-3, 126-128

21-4, 8-11

23-1, 104-107

23-1, 54-56

24-2, 20-23

24-3, 63-66

25-1, 45-47

25-2, 20-22

26-3, 95-97

27-2, 141-146

28-1, 64-66
0.8
0.9

2.7

2.7

155.3

155.3

$268.3\}$

$269.2\}$

391.8

393.3 .

448.6

$450.0\}$

469.3

486.5

495.9

515.4

524.7

$525.2\}$

533.6

553.3

562.9

571.8

573.6

583.1

584.4

587.4

609.8

610.5

613.6

630.6

$638.4\}$

640.8

641.8

642.1

667.1

668.1

$686.7\}$

688.6

704.5

705.7

727.0

744.8

771.2 
TABLE 15 - Continued

\begin{tabular}{cc}
\hline $\begin{array}{c}\text { Sample } \\
\text { (Interval in cm) }\end{array}$ & $\begin{array}{c}\text { Depth Below } \\
\text { Sea Floor (m) }\end{array}$ \\
\hline $30-2,132-134$ & 830.3
\end{tabular}

Hole 317A

2-1, 22-24

$2-1,41-43$

3-1, 103-105

$3-1,128-130$

3-2, 25-27

3-2, 28-30

5-1, 93-95

6-2, 103-105

8-1, 90-92

$10-2,128-130$

$10-2,130-132$

$12-4,5-7$

13-2, 49-51

15-2, 53-55

$15-4,102-104$

$18-2,60-62$

$18-2,72-74$

20-3, 50-52

22-2, 84-86

22-3, 18-20

22-3, 86-88

$22-4,139-141$

24-4, 70-72

24-4, 107-109

24-5, 113-115

26-1, 107-109

26-2, 25-27

28-1, 134-138

30-1, 88-92

$33-3,0-4$

\section{Hole 317B}

$1-1,20-22$

$1-1,22-24$

1-1, 101-102

1-2, 102-104

1-2, 29-31

$1-6,100-102$

$1-6,102-104$

4-2, 40-42

4-2, 43-45

4-3, 82-84

4-3, 85-87

8-2, 70-72

8-2, 73-75

$8-4,60-62$

8-4, 63-65

$12-1,70-72$

12-1, 73-75

$12-3,40-42$

$12-3,43-45$

$12-5,60-62$

$12-5,63-65$

16-1, 134-136

$16-1,136-138$

$16-2,60-62$

16-2, 62-64

16-4, 100-102

$16-4,103-105$

$16-6,60-62$

$16-6,62-64$

20-2, 53-55

20-2, 98-100

$20-4,58-60$

20-4, 60-62

20-6, 78-80

20-6, 81-83
$554.2\}$

554.4

564.5

564.8

565.3

565.3

576.9

585.0

602.4

623.3

644.1

651.0

$670.0\}$

673.5

698.6

$698.7\}$

727.0

755.9

756.7

757.4

759.4

777.7

778.1

779.6

821.1

821.8

849.9

887.4

927.5

$\left.\left.\begin{array}{r}0.3 \\ 0.3 \\ 1.1 \\ 1.1 \\ 1.9 \\ 8.6 \\ 8.6\end{array}\right\} \begin{array}{r}27.4 \\ 27.4 \\ 29.3 \\ 29.4 \\ 65.7 \\ 65.7 \\ 68.6 \\ 68.6 \\ 102.2 \\ 102.2 \\ 104.9 \\ 104.9 \\ 108.1 \\ 108.1 \\ 141.2 \\ 141.2 \\ 141.9 \\ 141.9 \\ 145.3 \\ 145.3 \\ 147.9 \\ 147.9 \\ 179.5 \\ 180.0 \\ 182.6 \\ 182.6 \\ 185.8 \\ 185.8\end{array}\right\}$

TABLE 15 - Continued

\begin{tabular}{|c|c|}
\hline $\begin{array}{c}\text { Sample } \\
\text { (Interval in } \mathrm{cm} \text { ) }\end{array}$ & $\begin{array}{l}\text { Depth Below } \\
\text { Sea Floor }(\mathrm{m})\end{array}$ \\
\hline $24-4,70-90$ & 220.7 \\
\hline $25-2,54-56$ & 227.1 \\
\hline $25-2,56-58$ & 227.1 \\
\hline $25-4,38-40$ & 229.9 \\
\hline $25-4,52-54$ & 230.0 \\
\hline $25-6,30-32$ & 232.8 \\
\hline $25-6,48-50$ & 233.0 \\
\hline $29-3,80-82$ & 266.8 \\
\hline $29-3,82-84$ & 266.8 \\
\hline $29-5,5-7$ & 269.1 \\
\hline $29-5,13-15$ & 269.1 \\
\hline $34-2,40-42$ & 312.4 \\
\hline $34-2,43-45$ & 312.4 \\
\hline $34-5,76-78$ & 317.3 \\
\hline $34-5,86-88$ & 317.4 \\
\hline $38-3,79-81$ & 352.3 \\
\hline $38-3,82-84$ & 352.3 \\
\hline $38-4,90-92$ & 353.9 \\
\hline $38-4,93-95$ & 353.9 \\
\hline $42-2,140-142$ & 389.4 \\
\hline \multicolumn{2}{|l|}{ Site 318} \\
\hline $1-1,129-132$ & 1.3 \\
\hline $1-2,82-85$ & 2.3 \\
\hline $1-3,65-70$ & 3.7 \\
\hline $1-3,72-78$ & 3.7 \\
\hline $1-4,65-72$ & 5.2 \\
\hline $14,72-76$ & 5.2 \\
\hline $1-5,94-103$ & 7.0 \\
\hline $4-1,41-43$ & 93.7 \\
\hline $4-1,45-47$ & 93.8 \\
\hline $4-2,71-73$ & 95.5 \\
\hline $4-2,74-76$ & 95.6 \\
\hline $4-3,72-74$ & 97.0 \\
\hline $4-3,74-76$ & 97.1 \\
\hline $4-4,70-72$ & 98.5 \\
\hline $4-4,7476$ & 98.6 \\
\hline $4-5,52-54$ & 99.8 \\
\hline $4-5,56-58$ & 99.9 \\
\hline $4-6,79-82$ & 101.6 \\
\hline $4-6,83-82$ & 101.6 \\
\hline $5-1,75-78$ & 122.5 \\
\hline $5-1,78-80$ & 123.9 \\
\hline $7-1,75-77$ & 179.8 \\
\hline $7-1,78-80$ & 179.8 \\
\hline $7-2,75-77$ & 181.3 \\
\hline $7-2,80-82$ & 181.3 \\
\hline $7-3,120-122$ & 183.2 \\
\hline $7-3,123-125$ & 183.2 \\
\hline $7-4,70-72$ & 184.2 \\
\hline $7-4,72-74$ & 184.2 \\
\hline $10-2,100-102$ & 266.5 \\
\hline $10-2,106-108$ & 266.6 \\
\hline $10-3,70-72$ & 267.7 \\
\hline $10-3,72-75$ & 267.7 \\
\hline $13-1,124-127$ & 350.8 \\
\hline $13-2,33-35$ & 351.3 \\
\hline $15-1,95-98$ & 407.5 \\
\hline $15-2,126-129$ & 409.3 \\
\hline $18-1,71-73$ & 492.7 \\
\hline $18-2,137-139$ & 494.9 \\
\hline $21-1,94-97$ & 578.5 \\
\hline $21-2,18-20$ & 579.2 \\
\hline $21-3,62-64$ & 581.1 \\
\hline $24-1,25-29$ & 615.8 \\
\hline $25-4,120-122$ & 630.7 \\
\hline $28-2,106-110$ & 665.6 \\
\hline $30-2,128-132$ & 703.8 \\
\hline $32-4,0-3$ & 743.5 \\
\hline
\end{tabular}

